\title{
An engineered, orthogonal auxin analog/ATIR1(F79G) pairing improves both specificity and efficacy of the auxin degradation system in Caenorhabditis elegans
}

Kelly Hills-Muckey ${ }^{1}$, Michael A. Q. Martinez ${ }^{2}$, Natalia Stec $^{1}$, Shilpa Hebbar $^{3}$, Joanne Saldanha ${ }^{2}$, Taylor N. Medwig-Kinney², Frances E. Q. Moore², Mariia Ivanova4; Ana Morao ${ }^{5}$, Jordan D. Ward $^{6}$, Eric G. Moss ${ }^{4}$, Sevinc Ercan ${ }^{5}$, Anna Y. Zinovyeva ${ }^{3}$, David Q. Matus ${ }^{2}$, Christopher M. Hammell $^{1, *}$

${ }^{1}$ Cold Spring Harbor Laboratory, 1 Bungtown Road, Cold Spring Harbor, NY 11724, USA

${ }^{2}$ Department of Biochemistry and Cell Biology, Stony Brook University, Stony Brook, NY 11794, USA

${ }^{3}$ Kansas State University, Division of Biology, Manhattan, KS 66506, USA.

${ }^{4}$ Department of Molecular Biology, Rowan University, Stratford, NJ 08084, USA.

${ }^{5}$ New York University, Center for Genomics and Systems Biology, 100 Washington Square

East, 852 Brown, New York, NY, 10003, USA.

${ }^{6}$ Department of Molecular, Cell, and Developmental Biology, University of California-Santa Cruz, Santa Cruz, CA 95064, USA

*Corresponding Author chammell@cshl.edu 


\section{ABSTRACT}

The auxin-inducible degradation system in C. elegans allows for spatial and temporal control of protein degradation via heterologous expression of a single Arabidopsis thaliana F-box protein, transport inhibitor response 1 (At TIR1). In this system, exogenous auxin (Indole-3-acetic acid; IAA) enhances the ability of ${ }_{A t} \mathrm{TIR} 1$ to function as a substrate recognition component that adapts engineered degron-tagged proteins to the endogenous $C$. elegans E3 ubiquitin ligases complex (SKR-1/2-CUL-1-F-box (SCF)), targeting them for degradation by the proteosome. While this system has been employed to dissect the developmental functions of many C. elegans proteins, we have found that several auxin-inducible degron (AID)-tagged proteins are constitutively degraded by ${ }_{A T}$ TIR1 in the absence of auxin, leading to undesired loss-of-function phenotypes. In this manuscript, we adapt an orthogonal auxin-derivative/mutant ${ }_{A t} \mathrm{TIR} 1$ pair (C. elegans AID version 2 (C.e.AIDv2)) that transforms the specificity of allosteric regulation of TIR1 from IAA to one that is dependent on an auxin derivative harboring a bulky aryl group (5-Ph-IAA). We find that a mutant ${ }_{A t} \mathrm{TIR}$ (F79G) allele that alters the ligand binding interface of TIR1 dramatically reduces ligand-independent degradation of multiple AID*-tagged proteins. In addition to solving the ectopic degradation problem for some AID targets, addition of 5-Ph-IAA to culture media of animals expressing ${ }_{A t}$ TIR1(F79G) leads to more penetrant loss-of-function phenotypes for AID*-tagged proteins than those elicited by the ${ }_{A t}$ TIR1-IAA pairing at similar auxin analog concentrations. The improved specificity and efficacy afforded by the mutant ${ }_{A t} \mathrm{TIR} 1(\mathrm{~F} 79 \mathrm{G})$ allele expands the utility of the AID system and broadens the number of proteins that can be effectively targeted with it. 


\section{ARITCLE SUMMARY}

Implementation of the auxin induced degradation (AID) system has increased the power if the $C$. elegans model through its ability to rapidly degrade target proteins in the presence of the plant hormone auxin (IAA). The current C.e.AID system is limited in that a substantial level of target degradation occurs in the absence of ligand and full levels of target protein degradation require high levels of auxin inducer. In this manuscript, we modify the AID system to solve these problems. 


\section{INTRODUCTION}

Detailed analyses of developmental and other dynamic biological events, processes and mechanisms have been facilitated in part by advancements in techniques to precisely control the products of gene expression. In Caenorhabditis elegans, a variety of tools have been developed to examine the stage- and tissue-specific function of genes, including FLP-mediated recombination to control gene activation(DAVIS et al. 2008; VoUTEV AND HUBBARD 2008) and RNAi to degrade the RNA gene product(QADOTA et al. 2007). While these techniques are indirect, limited by the stability of the target protein, and are prone to off-target effects, more modern approaches have leveraged the power of CRISPR genome editing to engineer gene products to harbor epitopes that enable proteins to be directly targeted for degradation.

The auxin-inducible degradation system allows for the rapid and conditional degradation of target proteins in yeast, vertebrate cells, and C. elegans (NISHIMURA et al. 2009; HOLLAND et al. 2012; ZHANG et al. 2015). This two-component system relies on the heterologous expression of a plant-specific F-box protein, TIR1, that binds the plant hormone auxin (RUEGGER et al. 1998; GRAY et al. 1999) and an encoded 44-amino acid minimal degron sequence (auxin-inducible degron $\left(\mathrm{AID}^{*}\right)$ ) derived from the Arabidopsis thaliana IAA17 protein(MORAWSKA AND ULRICH 2013). The tractability and specificity of this system to provide exquisite spatiotemporal control of target protein levels relies on two key features of the TIR1/AID*-tagged protein interaction. First, stable association of TIR1 with proteins harboring an AID* sequence is allosterically regulated by auxin (indole-3-acetic acid, or IAA)(TAN et al. 2007). Second, $A$. thaliana TIR1 can interact with endogenous members of the Skp1 and Cullin family of proteins to form a functional, ligand-gated ubiquitin E3 ligase that targets AID*-tagged proteins to the $26 \mathrm{~S}$ proteosome(NISHIMURA et al. 2009; KANKE et al. 2011; HOLLAND et al. 2012; ZHANG et al. 2015).

The AID system has proven to be extremely powerful in the $C$. elegans model where targeted inactivation of proteins can complement the already robust genetics of this system. The utility in this model comes from the application of many tissue-specific drivers that have been 
employed to drive TIR1 expression in various cell types(ASHLEY et al. 2021). Three issues have limited the applicability of this system for general use. First, the required dose of natural auxin (IAA) typically required for efficient degradation of target proteins is relatively high $(1 \mathrm{mM})$ and can elicit defined biological responses in C. elegans independent of ${ }_{A t} \mathrm{TIR} 1$ expression (BHOI et al. 2021; LOOSE AND GHAZI 2021). The current system also exhibits a substantial amount of activity against a simple, heterologous AID*::GFP reporter even in the absence of added IAA (MARTINEZ et al. 2020). Whether ectopic AtTIR1-mediated degradation is a general issue of the AID system or a reflection of latent properties of individual AID*-tagged target proteins in C. elegans is unknown. Alternatively, endogenously- or environmentally-derived partially activating ligands may be present in $C$. elegans and these metabolites may trigger aberrant AID-target degradation in the presence of ${ }_{A t}$ TIR1. Finally, some AID-targets are inefficiently degraded and fail to generate strong loss-of function phenotypes (PATEL AND HOBERT 2017; SERRANO-SAIZ et al. 2018; DUONG et al. 2020).

In this manuscript, we aimed to improve the $C$. elegans AID system by altering the ligand binding specificity of TIR1. Using a previously established TIR1 variant that can target degradation in A. thaliana using a synthetic, modified auxin/IAA ortholog (UCHIDA et al. 2018), we demonstrate that the ${ }_{A t} \operatorname{TIR} 1(\mathrm{~F} 79 \mathrm{G})$ variant combined with a 5-Ph-IAA auxin analog also functions in the $C$. elegans model to degrade AID*-tagged proteins. Importantly, substitution of this single amino acid in ${ }_{A t}$ TIR1 alleviates the ligand-independent activity of this protein for multiple, biologically relevant AID*-tagged fusion proteins. Finally, we demonstrate that the ${ }_{A t}$ TIR1(F79G)/5-Ph-IAA (C.e.AIDv2 System) also exhibits elevated activity, enabling strong loss-of-function phenotypes to be elicited with low levels of exogenously added ligand. Because the AIDv2 system efficiently targets existing AID*-tagged target genes, we suggest that this modification functions as an improved experimental platform to dissect spatial and temporal gene functions. 


\section{MATERIALS AND METHODS}

\section{C. elegans maintenance and genetics}

C. elegans strains were maintained on standard media at $20^{\circ} \mathrm{C}$ and fed E. coli OP50(BRENNER 1974). Some strains were provided by the CGC, which is funded by NIH Office of Research Infrastructure Programs (P40 OD010440). A complete list of strains outlined in this manuscript can be found in Supplemental Table 1. 400mM IAA (Sigma; Product \#I3750) and 100mM 5-PhIAA (Bioacademia; Product \#30-003-10) stocks were made in 95\% ethanol diluted into NGM media at the indicated concentration.

\section{CRISPR Editing}

cshls140(TIR1(F79G)): The single copy rps-28pro::AtTIR1::T2A::mCherry::his-11 (csh/s128) was integrated using standard CRISPR-mediated genomic editing to the ttTi5606 landing site following standard protocols(DICKINSON et al. 2013). CRISPR editing of the csh/s128 allele to generate the ${ }_{A t}$ TIR1(F79G) variant was accomplished using standard procedures (PAIX et al. 2017). Briefly, recombinant nlsCas9 obtained from the University of California, Berkley MacroLab was used in conjunction with a recombinant sgRNA (Synthego, Menlo Park, California) with the following guide sequence (PAM sequence underlined): TTCCCTTGAGCTCGACGGAACGG (Oligo \#1; see oligo table). A single stranded, HPLC-purified repair template (Oligo \#2; see oligo table) was used to edit the TIR1 coding sequence by homologous repair. The allele was verified by standard Sanger sequencing.

lin-28::AID*(ae157): The AID*-tagged lin-28 allele was constructed in a similar manner as above using a separate commercially available Cas9 protein (EnGen® Spy Cas9 NLS, M0646T) with gRNA targeting a genomic sequence in the $3^{\prime}$ coding sequence of lin-28 (5'ATATCATCGTCAGATGTAGT-3'). The sgRNA was synthesized using the Invitrogen ${ }^{\mathrm{TM}}$, MEGAshortscript ${ }^{\mathrm{TM}}$ T7 Transcription Kit (AM1354) and a single stranded transcription oligo \#1 
template (oligo \#3; see oligo table). The repair templates were generated using to template oligos \#4 and \#5 (see oligo table). Repair templates \#2 and \#3 were mixed together, heated to $96^{\circ} \mathrm{C}$ for 5 minutes, then placed on ice. The purpose was to create a "hybrid template" that is more efficient in the homology-directed repair(DOKSHIN et al. 2018).

ama-1::AID*::GFP(ers49): The $1752 \mathrm{bp}$ repair template to generate the AID*::GFP C-terminally tagged ama-1 allele was made by amplifying the AID*::GFP sequences from pLZ29(ZHANG et al. 2015) using the oligos \#6 and \#7 (see oligo table). Injection mixtures using recombinant $S$. pyogenes Cas9 3NLS (10 $\mu \mathrm{g} / \mu \mathrm{L}$, IDT), crRNA (GATGAATTTGGATCATAAGT, 2 nmol, IDT, oligo \#8; see oligo table), tracrRNA (IDT, cat\# 1072532) dsDNA donors, pCFJ90 (co-injection marker pharynx mCherry was used at a final concentration of $3 \mathrm{ng} / \mu \mathrm{L}$ ) were prepared as previously described(DOKSHIN et al. 2018).

hrpa-1::AID*(zen91): To generate hrpa-1::linker::AID*::TEV::FLAG strain, N2 worms were injected with the CRISPR-Cas9 RNA-protein complex(PAIX et al. 2017). The injection mix consisted of Alt-R Cas9 (IDT, cat\# 1081058) along with hrpa-1 crRNA (oligo \#9; see oligo table), dpy-10 crRNA (5'-GCUACCAUAGGCACCACGAG-3')(ARRIBERE et al. 2014), tracer RNA (IDT, cat\# 1072532) and hrpa-1::linker::AID*::TEV::FLAG donor (oligo \#10; see oligo table). Four independent alleles of hrpa-1::linker::AID*::TEV::FLAG were obtained and the tagged endogenous loci were sequenced. Two lines were chosen, outcrossed twice and assessed. One line was chosen for further experiments.

\section{Image acquisition}

Confocal Microscopy: Images were acquired using a Hamamatsu Orca EM-CCD camera and a Borealis-modified Yokagawa CSU-10 spinning disk confocal microscope (Nobska Imaging, Inc.) with a Plan-APOCHROMAT x $100 / 1.4$ or $40 / 1.4$ oil DIC objective controlled by MetaMorph 
software (version: 7.8.12.0). Animals were anesthetized on 5\% agarose pads containing 10mM sodium azide and secured with a coverslip. Imaging on the microfluidic device was performed on a Zeiss AXIO Observer.Z7 inverted microscope using a 40X glycerol immersion objective and DIC and GFP filters controlled by ZEN software (version 2.5). Images were captured using a Hamamatsu C11440 digital camera. For scoring plate level phenotypes, images were acquired using a Moticam CMOS (Motic) camera attached to a Zeiss dissecting microscope.

Wide-field Fluorescence microscopy: Images were acquired with a Zeiss Axio Observer microscope equipped with Nomarski and fluorescence optics as well as a Hamamatsu Orca Flash 4.0 FL Plus camera. An LED lamp emitting at $470 \mathrm{~nm}$ was used for fluorophore excitation. For single images, animals were immobilized on $2 \%$ agarose pads supplemented with $100 \mathrm{mM}$ Levamisole (Sigma).

\section{Image processing and analysis}

All acquired images were processed using Fiji software (version: 2.0.0- rc-69/1.52p)(SCHINDELIN et al. 2012). To quantify VPC-specific AID*::GFP or AMA-1::AID*::GFP expression levels, images were captured at the P6.p 1-cell stage (early L3 stage). Images of AID*::GFP animals were obtained at time points $0,30,60,90$, and 120 minutes in the absence or presence of auxin, and images of AMA-1::AID*::GFP animals were obtained 2 hours post-treatment. Expression levels were quantified by measuring the mean fluorescence intensity (MFI) of VPCs subtracted by the MFI of a background region in the image to account for camera noise. Cells were outlined using the freehand selection or wand (tracing) tool in Fiji. Kinetic data from AID*::GFP animals were normalized by dividing the MFI in treated or untreated animals at time points $30,60,90$, and 120 minutes by the average MFI in untreated animals at 0 minutes.

\section{Embryonic viability and brood size measurements}


Brood size measurements were carried out at $20^{\circ} \mathrm{C}$ and $25^{\circ} \mathrm{C}$ for each indicated strain using standard protocols (TISSENBAUM AND RUVKUN 1998). Briefly, single, late L4-stage animals of each genotype were transferred to a new plate every 24 hours for a total of four days. Plates were scored 24 and 48 hours after each transfer for the number of eggs and number of viable, hatched worms. The embryonic viability from each animal was established by calculating the percentage of unhatched eggs after 48 hours for each associated plate in the experiment. The total brood size was determined for individual worms by summing the number of viable offspring of each derived plate. In all cases the stated brood size is the average of $>15$ worms for each genotype. For hrpa-1::linker::AID*::TEV::FLAG brood measurements, embryos of each genotype were plated onto control, IAA, or 5-ph IAA plates and cultured until L4 stage. Single, late L4-stage animals of each genotype were transferred to a new plate. Larval arrest from each animal was established by calculating the percentage of arrested larvae that failed to grow after 24 hours.

\section{Motility assays}

Motility assays are modified from a previously published protocol (TSALIK et al. 2003). Briefly, 5 animals (grown in the indicated conditions) were transferred to a standard NGM plate ( \pm auxin analog) to a region in the center of a set of concentric rings $(4 \mathrm{~mm}, 6 \mathrm{~mm}, 8 \mathrm{~mm}, 10 \mathrm{~mm})(\mathrm{see}$ Template 1). Animals were allowed to freely move for the indicated time and the proportion of animals that left the first ring within the annotated time were recorded. Animals were scored positive even if they left the ring and returned within the boundary.

\section{Western blotting}

$40-50$ worms were picked directly into the $2 x$ Laemmli protein loading buffer and boiled at $95^{\circ} \mathrm{C}$ for 5 minutes. HRPA-1 was detected using the Monoclonal ANTI-FLAG M2-Peroxidase (HRP) antibody (A8592-2MG) from Sigma-Aldrich at a 1:500 dilution. Mouse anti-tubulin antibody (Sigma-Aldrich) was used at a 1:5000 dilution to detect tubulin as a loading control. 


\section{Graph Plots and Statistical Analysis}

Plots and diagrams were generated using GraphPad Prism v9 (GraphPad Software, San Diego,

Ca). Statistical significance was determined using a two-tailed un- paired Student's t-test. $P<0.05$ was considered statistically significant. ${ }^{* * * *}$ indicates $P<0.0001$.

\section{Data availability}

Strains in Supplementary Table S1 are available through the Caenorhabditis Genetics Center. Other strains and plasmids can be requested directly from the authors. The data that support the findings of this study are available upon reasonable request. 


\section{RESULTS}

${ }_{A t}$ TIR1(WT) triggers loss-of-function phenotypes of a LIN-28::AID* allele in the absence of exogenously-added ligand

During post-embryonic development, genes in the heterochronic pathway control the sequence of stage-specific cell divisions and cell fate specification (ROUGVIE AND MOSS 2013). A key feature of this pathway is that transitions from one stage-specific pattern of development to the next are controlled by the sharp temporal downregulation of protein coding genes (ROUGVIE AND Moss 2013). The lin-28 gene encodes a highly conserved RNA-binding protein that functions to modulate miRNA processing, turnover, and activity; lin-28 is acutely down-regulated after the first larval stage (MOSS et al. 1997; LEHRBACH et al. 2009; VAN WYNSBERGHE et al. 2011). lin-28 null mutants, lin-28(ga94), initiate post-embryonic development normally but then skip L2-stage patterns of cell division and immediately execute L3 patterns of development after the L1 molt (Fig. 1A) (Moss et al. 1997). Consequently, lin-28(0) mutants exhibit vulval morphology defects (protruding vulva; $p v l$ ), fail to proliferate their lateral seam cells, and precociously deposit adultspecific alae structures one stage earlier than wild-type animals (Figure 1B, Table 1).

To generate a lin-28 allele whose expression can be modulated by auxin, we used

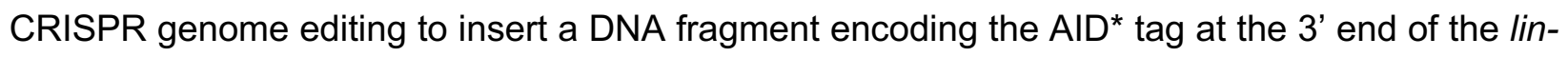
28 open reading frame. Examination of transgenic animals indicate that, in contrast to lin-28(ga54) animals, lin-28::AID*(ae127) animals do not exhibit heterochronic phenotypes, indicating that addition of the degron tag does not appreciably alter lin-28 activity (Figure 2B and Table 1). We next crossed the lin-28::AID*(ae127) allele into a strain harboring a ubiquitously expressed AtTIR1 (csh/s128 [rps-28pro::TIR1::T2A::mCherry::his-11]). Surprisingly, homozygous lin28::AID*(ae127); chs/s128 animals exhibited highly penetrant heterochronic phenotypes (precocious vulval cell divisions, altered seam cell lineage (seam cell number and the precocious production of adult alae structures at the L4 stage) on normal NGM plates indicating that that the 
TIR1 activity, in the absence of auxin, results in a reduction of lin-28 activity during development that phenocopies lin-28(If) phenotypes.

\section{Generation, validation, and characterization of an orthogonal auxin and ${ }_{A t}$ TIR1(F79G) allele in C. elegans}

Crystallographic studies indicate that IAA fills a hydrophobic cavity in between TIR1 and the AID/degron peptide sequence to form a stable trimeric complex (DHARMASIRI et al. 2005; KEPINSKI AND LEYSER 2005; TAN et al. 2007). We hypothesized that the TIR1-dependent/auxinindependent degradation phenotypes of the lin-28::AID* allele could be caused either by a lowlevel interaction between the AID*-tag and TIR1 in the absence of auxin or by the inappropriate recognition of chemically-related endogenously produced ligand that may bind to the pocket of ${ }_{A t}$ TIR1. Uchida et al. (UCHIDA et al. 2018) have previously described a series of mutations of TIR1 that alter the auxin binding pocket to enable ligand-dependent target degradation using IAA derivatives that have been modified by aryl groups on the $5^{\text {th }}$ position of IAA. One of the engineered ${ }_{A t}$ TIR1 mutants they identified, ${ }_{A t} \mathrm{TIR}$ (F79G), failed to interact with $\mathrm{AID}^{*}$-tagged proteins in the presence of IAA but exhibited high binding specificity for AID*-tagged substrates in the presence of 5-phenyl-indole-3-acetic acid (5-Ph-IAA)(UCHIDA et al. 2018). We reasoned that the inappropriate activity of ${ }_{A t} \mathrm{TIR} 1$, mediated by either of the above mechanisms, may be alleviated by similar alterations in the ${ }_{A t}$ TIR1-IAA binding pocket. A similar, orthogonal TIR1/auxin analog approach has been adapted for other systems using Oryza sativa TIR1 (OsTIR1) suggesting that this strategy is generally applicable (YESBOLATOVA et al. 2020).

To test the activity of a ${ }_{A t} T I R 1(F 79 G)$ allele of TIR1 in C. elegans, we used CRISPR genome editing and homology-directed repair (HDR) to edit an existing, single copy TIR1 allele. Specifically, animals harboring a ubiquitously expressed rps-28pro::TIR1 allele (csh/s128) and an eft-3pro::AID*::GFP transgene (ieSi58) were injected with a recombinant nlsCas9/guide ribonucleoprotein complex that is predicted to induce dsDNA breaks approximately $30 \mathrm{bp}$ 
upstream of the TIR1 F79 codon and a HRD repair oligo partially complementary to the TIR1 ORF overlapping with the TIR1 F79 codon (Figure 2). F1 progeny were then cloned onto NGM plates containing $1 \mathrm{mM}$ IAA to identify GFP(+) F2 progeny that fail to efficiently degrade the AID*::GFP reporter. Candidate GFP(+) F2 animals were then plated on NGM plates containing 100 $\mathrm{MM}$ 5-PhIAA to identify clonal animals harboring a modified ${ }_{A t}$ TIR1 allele that is capable of degrading the AID*::GFP reporter only in the presence of the 5-Ph-modified auxin (Figure 2B). A single recombinant ${ }_{A t} T I R 1(F 79 G)$ allele (csh/s140) was sequence-verified and used in further experiments.

We next compared the relative expression levels and degradation kinetics of the AID*::GFP reporter in animals expressing the two ${ }_{A t} \mathrm{TIR} 1$ variants. Previous observations indicate that ${ }_{A t}$ TIR1(WT) reduces AID*::GFP expression in the absence of IAA (MARTINEZ et al. 2020). We then compared the expression levels of the AID*::GFP reporter in animals expressing the two different TIR1 alleles. As shown in Figure 2C and E, AID*::GFP expression is significantly lower in the P6.p vulval precursor cells of animals expressing ${ }_{A t} \mathrm{TIR} 1(\mathrm{WT})$ when compared to the levels in similarly staged ${ }_{A t} \mathrm{TIR} 1(\mathrm{~F} 79 \mathrm{G})$-expressing animals. Expression levels of $\mathrm{AID}^{*}:: \mathrm{GFP}$ in ${ }_{A t}$ TIR1(F79G) animals is similar to that observed in WT animals (Figure S1). We next compared the efficiency of ligand-induced degradation mediated by these two ${ }_{A t} T I R 1$ alleles by quantifying the relative changes in $\mathrm{AID}^{*}:$ :GFP expression in the presence of various IAA or 5-Ph-IAA concentrations over time. We monitored AID*::GFP expression in single vulval precursor cells of stage-matched (early mid-L3 stage) animals that have been exposed to different concentrations of auxin orthologs that were incorporated into standard C. elegans solid culture media (Figure 2C and $\mathrm{D})$. We found that elevated concentrations $(\geq 0.05 \mathrm{mM})$ of both auxin analogs led to the efficient reduction of AID*::GFP with greater than $50 \%$ of the target protein depleted in P6.p cells within the first 30 minutes of the time course (Figure 2D). At lower concentrations of 5-Ph-IAA (0.05mM), At TIR1(F79G) maintained similar AID*::GFP depletion kinetics suggesting that this level 
of activating ligand is still saturating in this system and that overall kinetics are dependent on ${ }_{A t}$ TIR1(F79G) expression levels. In contrast, the efficiency of the ${ }_{A t}$ TIR1(WT)-mediated degradation when normal IAA was reduced (Figure 2D). These results suggest that the ${ }_{A t}$ TIR1(F79G) (AIDv2) functions more efficiently with the 5-Ph-IAA ligand than the classical version of the AID system with unmodified IAA(ZHANG et al. 2015).

\section{The AIDv2/TIR1(F79G) version of the AID system efficiently regulates the expression of dosage-sensitive AID* targets}

Given that the ${ }_{A t} \operatorname{TIR} 1(\mathrm{~F} 79 \mathrm{G})$ allele of ${ }_{A t} \mathrm{TIR} 1$ does not lead to a significant reduction of AID $^{*}:$ GFP expression in the absence of ligand, we next tested whether this allele could be used to efficiently modulate the activity of AID*-tagged genes that exhibit dosage-sensitive phenotypes in a ligand-specific manner. First, we crossed the ${ }_{A t} T I R 1$ (F79G) allele into a strain harboring the lin-28::AID* degron-tagged allele. As opposed to the inappropriate lin-28(If) phenotypes that are caused by ${ }_{A t} T I R 1(\mathrm{WT})$, lin-28::AID*; csh/s140 (At TIR1(F79G)) animals exhibit completely wild-type seam cell lineage (seam cell number) and do not exhibit precocious alae formation or a protruding vulval phenotype (Figure 3, Table 1). Importantly, treatment of lin28::AID*; csh/s140 (AtTIR1(F79G)) parental animals with very low concentrations of 5-Ph-IAA $(1 \mu \mathrm{M})$ lead to highly penetrant heterochronic phenotypes for each developmental feature (seam cell number, alae, and vulval development) in F1 progeny indicating that the AIDv2 system can elicit fully penetrant lin-28(If) phenotypes. These phenotypes were not elicited when animals were grown on media containing 1000-fold higher concentration of normal auxin (IAA), demonstrating the sensitivity and specificity of the TIR1(F79G) allele.

We have also previously tagged the large, catalytic subunit of the C. elegans pol II complex, ama-1, with the AID epitope with the idea that an auxin degradation system could be used to acutely inactivate transcription in a temporal and cell-type specific manner. This would 
bypass the limitations of prior approaches to reduce transcriptional output via chemical inhibitors or by cell-type-specific RNAi(RogALSKI et al. 1988; RogALSKI AND RIDDLE 1988; FIRNHABER AND HAMMARLUND 2013). Transgenic animals homozygous for a C-terminally tagged ama$1:: A I D^{*}:$ GFP allele, ama-1(ers49), are indistinguishable from wild-type animals and exhibit normal development and brood sizes (Figure 4A). When the ama-1::AID*::GFP allele is combined with a ubiquitously expressed ${ }_{A t} \mathrm{TIR} 1(\mathrm{WT})$, the pace of overall animal development is dramatically slowed and there is a significant reduction ( $>8$ fold) in the fecundity of animals compared to isogenic strains lacking ${ }_{A t} \mathrm{TIR} 1$ expression (Figure 4A). Consistent with the assumption that these developmental phenotypes result from an inappropriate activity of ${ }_{A t}$ TIR1(WT), AMA-1::AID*::GFP expression is reduced by approximately 2.9-fold in P6.p vulval precursor cells (1-cell stage) of animals expressing ubiquitous ${ }_{A t} \mathrm{TIR} 1(\mathrm{WT})$ when compared to a strain only expressing the tagged pol II allele (Figure 4B and C).

We next determined if the developmental phenotypes associated with the ectopic activity of ${ }_{A t} T I R 1(W T)$ would also be alleviated by mutating the binding pocket of the ${ }_{A t}$ TIR1 ligand binding domain. When we crossed the ama-1::AID*::GFP allele into a strain expressing ${ }_{A t} T I R 1(F 79 G)$, we found that animals exhibited normal brood sizes, consistent with a lack of ligand-independent activity for this TIR1 variant (Figure $4 A$ ). In addition, AMA-1::AID*::GFP expression levels in ${ }_{A t} T I R 1$ (F79G) expressing animals were similar to those observed in wild type (Figure 4B and C). Importantly, AMA-1::AID*::GFP expression is extinguished when animals are incubated with 5Ph-IAA, but not a 1000-fold higher concentration of regular IAA (Figure 4B and C). AMA$1::$ AID*::GFP depletion results in a variety of pleiotropic, terminal phenotypes (sterility, slow growth, arrest, and lethality) that resemble phenotypes associated with ama-1 mutations or animals that have been treated with alpha-amanitin, a pol II-specific enzymatic inhibitor (ROGALSKI et al. 1988; RogALSKI AND RIDDLE 1988; BIRD AND RIDDLE 1989). These results suggest that the 
${ }_{A t} T I R 1(F 79 G)$ allele of ${ }_{A t}$ TIR1 lacks appreciable ligand-independent activity on AID*-tagged substrates while maintaining the ability to program target degradation for multiple substrates.

\section{Exposure of $C$. elegans larva to high levels of IAA analogs elicits a transcriptional response}

Previous experiments have demonstrated that indole (and likely indole derivatives) derived from commensal bacteria extends lifespan (SONOWAL et al. 2017). Furthermore, recent reports utilizing the AID system have demonstrated that indole-3-acetic acid (IAA) at high physiological concentrations can also induce physiological responses in C. elegans that modulate a number of developmental and cellular activities. Specifically, continuous exposure of animals to auxin during development significantly extends the lifespan and can confer protection against endoplasmic reticulum (ER) stress (BHOI et al. 2021; LOOSE AND GHAZI 2021). Auxin antagonizes the negative effects of tunicamycin, a chemical inhibitor of $\mathrm{N}$-linked glycosylation and a known inducer of ER stress, and this protective response requires the activity of the XBP-IRE-1 pathway of the Unfolded Protein Response (UPR)(BHOI et al. 2021). These results collectively suggest that high levels of exogenous auxin may activate latent genetic programs that are optimized to ensure that animals can normally survive in diverse environments.

We discovered that auxin exposure induces the expression of multiple transcriptional reporters that are also induced in response to ER stress. These include transcriptional reporters for two glutathione S-transferases (gst-4 and gst-5) and gcs-1, encoding a gamma glutamylcysteine synthetase, that are upregulated by $\mathrm{SKN}-1 / \mathrm{Nrf}$, a transcription factor that orchestrates both oxidative and xenobiotic stress responses in $C$. elegans after exposure to tunicamycin (Figure 5A)(PAPP et al. 2012; GLOVER-CUTTER et al. 2013). The induction of this transcriptional response is not a generalized stress response as other transcriptional reporters of environmental stressors are not induced by auxin (e.g., hsp-16::GFP) (Figure 5A). Induction of gcs-1pro::GFP is rapid and peaks after 4-5 hours of $1 \mathrm{mM}$ auxin exposure (Figure $5 \mathrm{~B}$ ). This rapid and quantifiable response enabled us to both determine if 5-Ph-IAA also induces this regulatory pathway and then 
to identify the minimal dose of each auxin analog required to activate this gcs-1pro::GFP expression We found that both IAA and 5-ph-IAA induced gcs-1pro::GFP expression, but this induction was not significant over background until the auxin analog reached a concentration of greater than $0.5 \mathrm{mM}$ (Figure 5C). Therefore, an optimal AID system should work at exogenously added auxin analog concentrations that, at a minimum, do not trigger this transcriptional response.

The AIDv2 degradation system generates more penetrant loss-of-function phenotypes at lower IAA analog concentrations when compared to the classical TIR1(WT)-IAA pairing.

Given that the typical high levels of IAA or 5-Ph-IAA trigger the ectopic expression of gcs1pro::GFP, we aimed to determine if ${ }_{A t}$ TIR1(F79G) worked more efficiently than TIR1(WT) at lower auxin analog concentrations. Kinetic data comparing the two TIR1-auxin analog pairings in Figure 2C suggested that ${ }_{A t} T I R 1(F 79 G)$ more efficiently depletes the expression of $A D^{*}:: G F P$ than ${ }_{A t}$ TIR1(WT) in low auxin analog concentrations. To test this feature, we assayed both TIR1 variants for their ability to generate phenotypes on AID* targets that only display partially penetrant phenotypes with the AIDv1/TIR1(WT) system.

The unc-3 gene, encoding the sole C. elegans ortholog of the Collier/Olf/Ebf (COE) family of TFs, functions as a neuronal terminal selector gene that is required to both establish and maintain distinct cholinergic motor neuron cell fates in the ventral nerve chord (KERK et al. 2017; FENG et al. 2020; Ll et al. 2020). Animals harboring non-functional alleles of unc-3 display penetrant locomotion defects and exhibit a "coiler" phenotype where the posterior portions of the animal appear paralyzed (Figure 6A) (BRENNER 1974). To determine if ${ }_{A t}$ TIR1(F79G) can elicit more penetrant phenotypes than ${ }_{A t} \mathrm{TIR} 1(\mathrm{WT})$ at similar auxin analog concentrations, we scored uncoordinated phenotypes of ${ }_{A t}$ TIR1(F79G) or AtTIR1(WT) parental animals exposed to varied auxin analog concentrations using a modified radial motility assay (TSALIK et al. 2003) (Figure 6B). Specifically, late L4/adult F1 animals that had or had not been exposed to auxin analogs 
were transferred to a center point of a series of concentric circles of a marked NGM plate. Animals were then allowed to move freely for 2 minutes and the percentage of animals that moved greater than $2 \mathrm{~mm}$ from that center point were scored (Figure 6C). Using this assay, 100\% of wild-type and $\sim 2 \%$ of unc-3(e151) animals moved $>2 \mathrm{~mm}$ within the recorded time course (Figure $6 \mathrm{C})$. We found that treatment of ${ }_{A t} T I R 1(W T) ; \quad u n c-3:: m N G:: A I D^{*}$ animals with IAA induced an uncoordinated phenotype but only at elevated IAA concentrations (approximately $45 \%$ of animals were motile enough to travel outside of the assay area (Figure 6C). In contrast, 5-Ph-IAA treatment of ${ }_{A t} \operatorname{TIR} 1(\mathrm{~F} 79 \mathrm{G})$ animals led to highly penetrant unc phenotypes in this assay (Figure 6C). Consistent with unc-3 activity being continuously required for normal motility, At TIR1(F79G) animals treated after hatching also developed an unc phenotype by young adulthood, though the penetrance of these phenotypes was reduced when compared to continuous 5-Ph-IAA treatment (Figure S1). We do note that the expressivity of the unc-3 phenotypes elicited by 5-Ph-IAA treatment in ${ }_{A t} T I R 1(F 79 G) ;$ unc-3::GFP::AID* animals, as measured by the proportion of animals that exhibiting a "coiler" phenotype, is lower than what is observed for strong unc-3 loss-offunction alleles and are consistent with residual unc-3 activity in 5-Ph-IAA treated animals.

Genes encoding essential, ubiquitously expressed RNA binding proteins are typically difficult to study using standard genetics or RNAi because maternal contribution and partial rescue of these highly expressed genes confounds analyses, and for RNAi, the half-life of these proteins is typically long. An example of this class of gene is the C. elegans ortholog of HNRNPA2, hrpa-1 (RYAN AND HART 2021; RYAN et al. 2021). Null mutations of hrpa-1, hrpa-1(ok963), can be propagated in balanced strains and homozygous hrpa-1(ok963) animals segregating from these animals exhibit a dramatic reduction in brood size and the resulting F1 progeny of these animals exhibit a fully penetrant larval lethal phenotype (RYAN AND HART 2021). Homozygous animals expressing a CRISPR-engineered, AID*-tagged version of hrpa-1, hrpa-1(zen91), exhibit a wild type growth phenotype indicating that the AID* tag does not interfere with normal development. Consistent with previous AID*-tagged proteins, HRPA-1::AID*::TEV::FLAG animals expressing 
At TIR1(WT) exhibit a reduction in expression of the AID*-tagged target in the absence of IAA, when compared to animals expressing the ${ }_{A t} T I R 1$ (F79G) allele (Figure 7A and B). We next used the hrpa-1::AID*::TEV::FLAG allele to determine if the C.e.AIDv2 system was more efficient at degrading target proteins than the standard ${ }_{A t} \mathrm{TIR} 1(\mathrm{WT})$ variant. To do this, we hatched transgenic animals on solid NGM media containing either IAA or 5-Ph-IAA (at the indicated concentration) and allowed these animals to grow to a young adult stage of development. Quantitative westerns ( $n=4)$ indicate that both TIR1 variants strongly deplete HRPA-1::AID*::TEV::FLAG. We next calculated the fold reduction of HRPA-1::AID*::TEV::FLAG expression in each case and determined that the C.e.AIDv2 system was reproducibly more effective at depleting the AID*target protein regardless of auxin analog concentration (Figure 7C). Treatment of ${ }_{A t} T I R 1(F 79 G)$; hrpa-1::AID*::TEV::FLAG with 5-Ph-IAA lead to phenotypes that superficially resemble phenotypes exhibited by homozygous hrpa-1(ok963) animals (Figure 7D). To compare the TIR1 two variants at the phenotypic level, we quantified brood size and percent of arrested F1 broods. Consistent with a strong depletion of $h r p a-1:: A I D^{*}:: T E V:: F L A G$ by both TIR1 alleles, addition of auxin analogs to the growth media strongly affected brood sizes (Figure 7E). In contrast to the nonpenetrant $\mathrm{F} 1$ arrest phenotypes of ${ }_{A t} T I R 1(W T)$ animals on IAA-containing media, treatment of AtTIR1(F79G); HRPA-1::AID*::TEV::FLAG animals with 5-Ph-IAA lead to a fully penetrant F1 embryonic or larval arrest phenotype that is identical to those measured in homozygous hrpa1(ok963) animals (Figure 7F). We conclude that the C.e.AIDv2 version of the auxin degradation system more efficiently depletes AID*-tagged target proteins. 


\section{DISCUSSION}

The ability to rapidly and conditionally deplete target proteins using the heterologous TIR1 degradation system has dramatically improved the utility of the $C$. elegans model for dissecting aspects of multicellular development (ZHANG et al. 2015). This is in part due to the relatively modular structure of the system which requires a single genetic modification of a target gene to insert the AID* epitope and the heterologous expression of a single E3 adaptor protein, TIR1, that, in response to the addition of an auxin analog, rapidly targets the AID*-tagged protein for degradation. This approach has enabled many gene products that were refractory to genetic manipulation to be studied in detail. In the intervening six years from its first description in $C$. elegans, the number of AID-tagged genes that have already been generated is estimated to be in the hundreds with more than 20 currently available at the C. elegans Genome Stock Center (CGC). For a small number of target genes, two features of the original system still exist. First, the classical allele of TIR1 exhibits a level of activity in the absence of added auxin/IAA ligand that inappropriately reduces target gene expression. As outlined in this manuscript, this ligandindependent activity is substantial and can range from $20 \%$ (for AID*::GFP and HRPA-1::AID*) to $65 \%$ (in the case of AMA-1::AID*::GFP). Relatively mild reductions of AID*-tagged proteins may not be physiologically important for many AID* targets but for other important developmental genes, this off-target activity makes the classic AID system unusable. Second, we and others find that auxin/IAA concentrations typically used to inactivate AID* target genes in C. elegans induce a physiological response that may occlude or modulate phenotypic observations in as of yet, unanticipated ways (BHOI et al. 2021; LOOSE AND GHAZI 2021). Our analysis of gcs-1pro::GFP expression indicates that at least one of these responses can occur at levels above 0.5mM IAA and are independent of TIR1 expression.

In this manuscript, we describe a modification to the TIR1 protein that solves both of these problems. Specifically, mutation of the phenylalanine 79 to a glycine residue prevents ectopic TIR1 activity while maintaining the ability to regulate AID*-tagged gene destruction through the 
addition of a synthetic IAA analog, 5-Ph-IAA. Further analysis of the activity of the ${ }_{A t}$ TIR1(F79G) variant also indicates that it exhibits a fortuitous increase in relative activity that generates more penetrant loss-of-function phenotypes for several AID*-tagged genes than those elicited with the classical TIR1 (unc-3 and hrpa-1). This enables experiments to be performed at auxin analog concentrations that do not elicit undesired phenotypic consequences. The reduction in off-target effects and increase in activity can arise from a number of biochemical features that could be altered by modifying the ligand binding pocket of TIR1. We hypothesize that the inappropriate degradation of AID*-targets that occurs without the addition of exogenously added auxin may be facilitated by auxin-related indoles, whose origin may be dependent on the $E$. coli bacterial food source. It is known that commensal bacteria provide indole-related compounds to developing larva and these indoles induce phenotypic changes(SONOWAL et al. 2017). Furthermore, we favor this hypothesis because we have noted that several sensitive AID*-tagged genes exhibit variably penetrant phenotypes that track proportionally with the age of the bacterial lawn used to culture animals. By altering the binding pocket of TIR1, the TIR1(F79G) variant may no longer be able to bind these food-derived indoles. The TIR1(F79G) variant would then be activated exclusively by the engineered 5-Ph-IAA ligand leading to enhanced experimental efficacy and tractability.

An important feature of the C.e.AIDv2 system described here is that previously existing AID*-tagged genes remain targetable with this system. This feature can be exploited in two ways. Using the CRISPR guide and repair template we used to generate the initial F79G variant, any other single copy TIR1(WT) transgene derived from the original TIR1 sequences described in Zhang et al. can be easily engineered to express the 5-Ph-IAA inducible variant, including the recent array of tissue specific drivers described in Ashley et al 2001 (Figure 2A)(ZHANG et al. 2015; ASHLEY et al. 2021; Vo et al. 2021). Alternatively, we have engineered the ubiquitously expressed rps-28pro::TIR1(F79G) allele to contain unique, CRISPR targetable sites that flank the rps-28 promoter sequences that can be utilized for genomic engineering and promoter preplacement. In this case, alternative promoters can easily be exchanged by homology-directed 
repair (HDR) (Figure S3). This method has the added advantage in that the TIR1(F79G) allele expressed from the single copy cshls140 allele also expresses a highly visible mCherry::HIS-11 reporter, whose ubiquitous expression will change to the expression pattern programed by any recombinant promoter sequences.

Finally, this engineered system is ripe for further enhancement at the molecular/genetic and chemical levels. In other heterologous systems, TIR1 has been modified to contain a nuclear localization sequence that targets TIR1 activity to the nucleus. This modification increases the ability of TIR1 to degrade several, high abundance nuclear proteins (KANKE et al. 2011). Fusion of a Skp1 ortholog, a component of the SCF complex, to TIR1 also improves degradation kinetics and loss-of-function phenotypes of AID*-tagged target genes that are refractory to TIR1 alone suggesting that these additions may also improve the TIR1(F79G) activity in C. elegans. Finally, 5-Ph-IAA does not appear to penetrate the eggshell. Previously described chemical modifications of IAA increase have been demonstrated to alleviate this limitation, enabling more sophisticated temporal studies of developmental processes to be addressed. It seems likely that similar modifications to 5-Ph-IAA may improve the activity of the C.e.AIDv2 system. 


\section{ACKNOWLEDGEMENTS}

We would like to thank members of the Hammell and Matus laboratories for critical review of this manuscript. We received strains from Oliver Hobert and from the Caenorhabditis Genetics Center (CGC), which is funded by NIH Office of Research Infrastructure Programs (P40 OD010440).

\section{FUNDING}

DQM is funded by the NIH NIGMS (R01GM121597) and the Damon Runyon Cancer Research Foundation (DRR-47-17). AYZ is supported by the NIH NIGMS (R35GM124828). MAQM was supported by an NIH NIGMS Diversity Supplement (R01GM121597) and is currently funded by the $\mathrm{NIH} \mathrm{NCI} \mathrm{(F30CA257383).} \mathrm{TNM-K} \mathrm{is} \mathrm{supported} \mathrm{by} \mathrm{the} \mathrm{NIH} \mathrm{NICHD} \mathrm{(F31HD100091).} \mathrm{FEQM} \mathrm{is}$ supported by an NIH NIGMS Diversity Supplement (R01GM121597). JDW is supported by the NIH NIGMS (R01GM138701). SE and AM were supported by NIH NIGMS R35 GM130311. Cold Spring Harbor Laboratory, the Rita Allen Foundation, and NIH NIGMS (R01GM117406) support $\mathrm{CMH}$.

\section{CONFLICTS OF INTEREST}

None declared. 
Figure Legends:

Figure 1. AtTIR1(WT) induces a lin-28 loss-of-function phenotype in the absence of exogenously added auxin ligand. (A) The lateral seam cells of wild-type C. elegans larva exhibit a stereotyped cell division program that generates additional seam cell in the L2 stage. Mutations in the heterochronic gene lin-28 result in an altered seam cell division pattern due to the skipping of L2 stage-specific developmental programs. In addition, the lateral seam cells lin-28(0) mutants precociously exit the cell cycle and inappropriately deposit alae, adult-specific cuticle structures, during the L3-L4 molt. (B) In contrast to the normal skin and vulval developmental programs of wild-type animals and animals that harbor an AID-tagged lin-28 allele, lin-28(0) mutants exhibit precocious alae at the L4 stage and a protruding vulval phenotype (pvl). (C) Combining the AIDtagged lin-28 allele with a ubiquitously expressed AtTIR1(WT) allele results in strong heterochronic phenotypes in the absence of additional auxin. Dashed yellow lines indicate the absence of adult stage-specific alae structures whereas a solid line demarcates the presence of adult stage-specific alae structures. (See Table 1 for details).

Figure 2. Mutation of phenylalanine 79 to glycine in the TIR1 protein switches the specificity of the auxin degradation system to one that is now responsive to 5-Ph-IAA. (A) Structure of cshls128 that encodes both a ${ }_{A t}$ TIR1(WT) protein and an autocatalytically-cleaved nuclear localized mCherry::HIS-11 reporter driven by a ubiquitously-expressed ribosomal protein (rps-28) promoter. Below the gene structure is the coding sequence of the region of ${ }_{A t} \mathrm{TIR} 1$ (WT) that was mutagenized via CRISPR and HDR using a co-injected ssDNA oligo. (B) Representative mid-larval staged animals expressing $A_{I D}^{*}::$ GFP and one of two indicated ${ }_{A t}$ TIR1 variants. Animals were grown continuously on untreated NGM plates or NGM plates including the indicated auxin analog at the listed concentration. (C) Micrographs of early L3 staged animals expressing AID*::GFP and one of the two ${ }_{A t} T I R 1$ variants before and 120 minutes after the addition of the auxin analog. (D) Rates of AID*::GFP degradation were determined by quantifying AID*::GFP in 
early L3 staged P6.p cells in animals co-expressing the indicated ${ }_{A t}$ TIR1 variant following auxin analog treatment. Data presented as the mean and SD ( $n \geq 10$ animals examined for each time point). (E) Quantification of the relative expression levels of the AID*::GFP reporter in P6.p cells of early L3 staged animals that were grown on control plates. Data presented as the median with SD $\left(n \geq 10\right.$ animals examined for each TIR1 transgene, and ${ }^{* * * *}=p<0.0001$ by a Mann Whitney U-test).

Figure 3. The ${ }_{A t}$ TIR1(F79G) variant of TIR1 allows for ligand-dependent regulation of LIN28::AID* activity by 5-PhIAA. Representative images depict the cuticular and vulval development at the L4-stage of F1 animals that have developed on NGM plates containing the auxin analog at the indicated concertation. Dashed yellow lines demarcate the absence of adult stage-specific alae structures whereas a solid line demarcates the presence of adult stagespecific alae structures. Pvl = protruding vulva. (See Table 1 for details).

Figure 4. ${ }_{A t} \mathrm{TIR} 1(\mathrm{WT})$ allele of TIR1 generates ligand-independent loss-of-function phenotypes that are alleviated by the 5-Ph-IAA regulated ${ }_{A t}$ TIR1(F79G) variant. (A) An AIDtagged, endogenous allele of ama-1, ama-1(ers49), exhibits a severe reduction in brood size when combined with ${ }_{A t}$ TIR1(WT) even in the absence of added auxin. In contrast, the ${ }_{A t}$ TIR1(F79G) variant does not alter the brood size of ama-1(ers49) animals ( $\mathrm{n}=15$ for each genotype and significance was calculated using an unpaired t-test). (B) The expression of AMA$1:: A I D^{*}::$ GFP is dramatically reduced in animals expressing ${ }_{A t}$ TIR1(WT) and not in animals expressing ${ }_{A t}$ TIR1(F79G). Shown are DIC and corresponding GFP images of VPCs (from early L3 stage animals at the P6.p 1-cell stage). Scale bar is 10 microns. (C) Quantification of AMA$1::$ AID*::GFP expression animals depicted in panel B. Data presented as the mean \pm SD $(n \geq 10$ animals examined for each, and $P<0.0001$ by a Student's t-test). (D) AMA-1::AID*::GFP is degraded in the germline by 5-Ph-IAA. Representative DIC and GFP images of L4 staged animals 
that have been transferred to control NGM plates or NGM plates containing $0.5 \mathrm{mM} 5$-Ph-IAA for 2 hours.

Figure 5. Elevated levels of auxin analogs induce stress-responsive gene expression. (A) Representative GFP images and estimation plots of the expression levels of each indicated stress-responsive reporter in animals that have been exposed to auxin/IAA for 4 hours. For quantification of GFP expression, the relative fluorescence of whole, individual animals was measured ( $n>13$ for each reporter). (B) Transgenic gcs-1pro::GFP animals were exposed to $1 \mathrm{mM}$ IAA for the indicated times and relative fluorescence of individual animals was calculated as in Panel A. (C) Quantification of gcs-1pro::GFP expression levels in animals that have been incubated on plates containing the indicated concentrations of IAA or 5-Ph-IAA for 4 hours.

Figure 6. The C.eAIDv2 system generates more penetrant uncoordinated phenotypes than the ${ }_{A t}$ TIR1(WT)/IAA pairing for an AID-tagged allele of UNC-3. (A) The plate phenotypes of wild-type, unc-3(e151), and ${ }_{A t} T I R 1(F 79 G) ;$ unc-3::GFP::AID animals \pm 5-Ph-IAA. Loss-offunctional alleles of unc-3 cause animals to exhibit the "coiler" phenotype in which the tails of animals are paralyzed and coiled. (B) Depiction of the modified motility assay in which animals are placed in the center of a defined region of a solid media plate and allowed to freely move for a defined period of time. Animals were scored as uncoordinated (unc) if they failed to move out of the prescribed circle. (C) Quantification of the animals in the modified mobility assay. Each dot represents the data from four separate experiments containing 5 animals per circle.

Figure 7. The C.eAIDv2 system phenocopies hrpa-1(0) phenotypes with a AID-tagged hrpa1 allele. (A) Quantitative western blots depicting the levels of HRPA-1::AID::TEV::FLAG in transgenic animals exposed to the indicated auxin analogs. For drug treatments, indicated animals were incubated from hatching to young adulthood with the indicated concentration of 
auxin analogs. (B) Quantification of the relative levels of HRPA-1::AID::TEV::FLAG in animals expressing ${ }_{A t} T I R 1(W T)$ or ${ }_{A t} T I R 1(F 79 G)$ on normal solid NGM media $(\mathrm{n}=4)$. (C) Calculation of the relative fold reduction in HRPA-1::AID::TEV::FLAG expression in ${ }_{A t} T I R 1(W T)$ or ${ }_{A t} T I R 1(F 79 G)$ expressing animals exposed to auxin or auxin analog. (D) Pictomicrographs of representative young adult $h r p a-1(0)$ animal or ${ }_{A t} T I R 1(F 79 G) ; ~ h r p a-1:: A I D:: T E V:: F L A G$ animals that have been

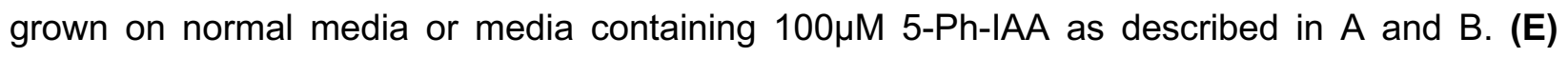
Quantification of the brood size of wild-type, $h r p 1-1(0)$ and animals expressing hrpa$1:: A I D:: T E V:: F L A G$ and one of the two TIR1 variants on or off the indicated auxin analogs. (F) Calculation of the percentage of broods with arrested F1 progeny.

\section{Table Legends}

Table 1. Measurement of TIR1- and ${ }_{A t}$ TIR1(F79G)-dependent heterochronic phenotypes in strains harboring an AID-tagged lin-28 allele. A comparison and quantification of adult alae, seam cell numbers and percent of animals that exhibit protruding vulva phenotypes for animals expressing an AID-tagged lin-28 allele.

\section{Supplemental Figures}

Figure S1. The relative expression levels of AID*::GFP (ieSi58) in animals expressing TIR1 variants. The relative expression levels of the AID*::GFP reporter were quantified by measuring the pixel volumes of identically sized regions of the terminal pharyngal bulbs of young adultstaged animals. Data presented as the median with SD ( $n=10$ animals examined for each TIR1 transgene, and ${ }^{* * *}=\mathrm{P}<0.0001$ by a Mann Whitney U-test).

Figure S2. The C.eAIDv2 system generates more penetrant uncoordinated phenotypes than the ${ }_{A t}$ TIR1(WT)/IAA pairing for an AID-tagged allele of unc-3 when animals are exposed 
to 5-Ph-IAA during pos-embryonic development. Quantification of the indicated animals in the modified mobility assay that have been exposed to 5-Ph-IAA from hatching to early adulthood. Each dot represents the data from four separate experiments containing 5 animals per circle.

Figure S3. Two CRISPR-mediated methodologies to adapt the C.e.AIDv2 system. (A) For existing TIR1 drivers based on the original Zhang et al. TIR1 coding sequences, a single sgRNA guide/ssDNA oligo conversion strategy can be employed to generate the TIR1(F79G) variant. Using the sg RNA and repair template outlined in the methods and oligos section, conversion of previous TIR1 alleles results in the deletion of a Sacl restriction site. Therefore, when successful editing has occurred, a PCR product that spans this region will lack the Sacl restriction site and can be used to identify successful edits by PCR. (B) Alternatively, two CRISPR guides can be used to remove the rps-28 promoter and HDR can be used to recombine in the promoter of interest. 


\section{BIBLIOGRHY}

Arribere, J. A., R. T. Bell, B. X. Fu, K. L. Artiles, P. S. Hartman et al., 2014 Efficient marker-free recovery of custom genetic modifications with CRISPR/Cas9 in Caenorhabditis elegans. Genetics 198: 837-846.

Ashley, G. E., T. Duong, M. T. Levenson, M. A. Q. Martinez, L. C. Johnson et al., 2021 An expanded auxin-inducible degron toolkit for Caenorhabditis elegans. Genetics 217.

Bhoi, A., F. Palladino and P. Fabrizio, 2021 Auxin confers protection against ER stress in Caenorhabditis elegans. Biol Open 10.

Bird, D. M., and D. L. Riddle, 1989 Molecular cloning and sequencing of ama-1, the gene encoding the largest subunit of Caenorhabditis elegans RNA polymerase II. Mol Cell Biol 9: 4119-4130.

Brenner, S., 1974 The genetics of Caenorhabditis elegans. Genetics 77: 71-94.

Davis, M. W., J. J. Morton, D. Carroll and E. M. Jorgensen, 2008 Gene activation using FLP recombinase in C. elegans. PLoS Genet 4: e1000028.

Dharmasiri, N., S. Dharmasiri and M. Estelle, 2005 The F-box protein TIR1 is an auxin receptor. Nature 435: 441-445.

Dickinson, D. J., J. D. Ward, D. J. Reiner and B. Goldstein, 2013 Engineering the Caenorhabditis elegans genome using Cas9-triggered homologous recombination. Nature methods 10: 1028-1034.

Dokshin, G. A., K. S. Ghanta, K. M. Piscopo and C. C. Mello, 2018 Robust Genome Editing with Short Single-Stranded and Long, Partially Single-Stranded DNA Donors in Caenorhabditis elegans. Genetics 210: 781-787.

Duong, T., N. R. Rasmussen, E. Ballato, F. S. Mote and D. J. Reiner, 2020 The Rheb-TORC1 signaling axis functions as a developmental checkpoint. Development 147.

Feng, W., Y. Li, P. Dao, J. Aburas, P. Islam et al., 2020 A terminal selector prevents a Hox transcriptional switch to safeguard motor neuron identity throughout life. Elife 9.

Firnhaber, C., and M. Hammarlund, 2013 Neuron-specific feeding RNAi in C. elegans and its use in a screen for essential genes required for GABA neuron function. PLoS Genet 9: e1003921.

Glover-Cutter, K. M., S. Lin and T. K. Blackwell, 2013 Integration of the unfolded protein and oxidative stress responses through SKN-1/Nrf. PLoS Genet 9: e1003701.

Gray, W. M., J. C. del Pozo, L. Walker, L. Hobbie, E. Risseeuw et al., 1999 Identification of an SCF ubiquitin-ligase complex required for auxin response in Arabidopsis thaliana. Genes Dev 13: 1678-1691.

Holland, A. J., D. Fachinetti, J. S. Han and D. W. Cleveland, 2012 Inducible, reversible system for the rapid and complete degradation of proteins in mammalian cells. Proceedings of the National Academy of Sciences of the United States of America 109: E3350-3357.

Kanke, M., K. Nishimura, M. Kanemaki, T. Kakimoto, T. S. Takahashi et al., 2011 Auxininducible protein depletion system in fission yeast. BMC cell biology 12: 8 .

Kepinski, S., and O. Leyser, 2005 The Arabidopsis F-box protein TIR1 is an auxin receptor. Nature 435: 446-451.

Kerk, S. Y., P. Kratsios, M. Hart, R. Mourao and O. Hobert, 2017 Diversification of C. elegans Motor Neuron Identity via Selective Effector Gene Repression. Neuron 93: 80-98.

Lehrbach, N. J., J. Armisen, H. L. Lightfoot, K. J. Murfitt, A. Bugaut et al., 2009 LIN-28 and the poly $(U)$ polymerase PUP-2 regulate let-7 microRNA processing in Caenorhabditis elegans. Nat Struct Mol Biol 16: 1016-1020.

Li, Y., A. Osuma, E. Correa, M. A. Okebalama, P. Dao et al., 2020 Establishment and maintenance of motor neuron identity via temporal modularity in terminal selector function. Elife 9 . 
Loose, J. A., and A. Ghazi, 2021 Auxin treatment increases lifespan in Caenorhabditis elegans. Biol Open 10.

Martinez, M. A. Q., B. A. Kinney, T. N. Medwig-Kinney, G. Ashley, J. M. Ragle et al., 2020 Rapid Degradation of Caenorhabditis elegans Proteins at Single-Cell Resolution with a Synthetic Auxin. G3 (Bethesda) 10: 267-280.

Morawska, M., and H. D. Ulrich, 2013 An expanded tool kit for the auxin-inducible degron system in budding yeast. Yeast 30: 341-351.

Moss, E. G., R. C. Lee and V. Ambros, 1997 The cold shock domain protein LIN-28 controls developmental timing in C. elegans and is regulated by the lin-4 RNA. Cell 88: 637-646.

Nishimura, K., T. Fukagawa, H. Takisawa, T. Kakimoto and M. Kanemaki, 2009 An auxin-based degron system for the rapid depletion of proteins in nonplant cells. Nat Methods 6: 917922.

Paix, A., A. Folkmann and G. Seydoux, 2017 Precision genome editing using CRISPR-Cas9 and linear repair templates in C. elegans. Methods 121-122: 86-93.

Papp, D., P. Csermely and C. Soti, 2012 A role for SKN-1/Nrf in pathogen resistance and immunosenescence in Caenorhabditis elegans. PLoS Pathog 8: e1002673.

Patel, T., and O. Hobert, 2017 Coordinated control of terminal differentiation and restriction of cellular plasticity. eLife 6: 249.

Qadota, H., M. Inoue, T. Hikita, M. Koppen, J. D. Hardin et al., 2007 Establishment of a tissuespecific RNAi system in C. elegans. Gene 400: 166-173.

Rogalski, T. M., A. M. Bullerjahn and D. L. Riddle, 1988 Lethal and amanitin-resistance mutations in the Caenorhabditis elegans ama-1 and ama-2 genes. Genetics 120: 409422.

Rogalski, T. M., and D. L. Riddle, 1988 A Caenorhabditis elegans RNA polymerase II gene, ama-1 IV, and nearby essential genes. Genetics 118: 61-74.

Rougvie, A. E., and E. G. Moss, 2013 Developmental Transitions in C. elegans Larval Stages. Curr Top Dev Biol 105: 153-180.

Ruegger, M., E. Dewey, W. M. Gray, L. Hobbie, J. Turner et al., 1998 The TIR1 protein of Arabidopsis functions in auxin response and is related to human SKP2 and yeast grr1p. Genes Dev 12: 198-207.

Ryan, V. H., and A. C. Hart, 2021 sym-2 loss-of-function causes glutamatergic neurodegeneration after oxidative stress. MicroPubl Biol 2021.

Ryan, V. H., T. M. Perdikari, M. T. Naik, C. F. Saueressig, J. Lins et al., 2021 Tyrosine phosphorylation regulates hnRNPA2 granule protein partitioning and reduces neurodegeneration. EMBO J 40: e105001.

Schindelin, J., I. Arganda-Carreras, E. Frise, V. Kaynig, M. Longair et al., 2012 Fiji: an opensource platform for biological-image analysis. Nature methods 9: 676-682.

Serrano-Saiz, E., E. Leyva-Diaz, E. De La Cruz and O. Hobert, 2018 BRN3-type POU Homeobox Genes Maintain the Identity of Mature Postmitotic Neurons in Nematodes and Mice. Curr Biol 28: 2813-2823 e2812.

Sonowal, R., A. Swimm, A. Sahoo, L. Luo, Y. Matsunaga et al., 2017 Indoles from commensal bacteria extend healthspan. Proc Natl Acad Sci U S A 114: E7506-E7515.

Tan, X., L. I. Calderon-Villalobos, M. Sharon, C. Zheng, C. V. Robinson et al., 2007 Mechanism of auxin perception by the TIR1 ubiquitin ligase. Nature 446: 640-645.

Tissenbaum, H. A., and G. Ruvkun, 1998 An insulin-like signaling pathway affects both longevity and reproduction in Caenorhabditis elegans. Genetics 148: 703-717.

Tsalik, E. L., T. Niacaris, A. S. Wenick, K. Pau, L. Avery et al., 2003 LIM homeobox genedependent expression of biogenic amine receptors in restricted regions of the $\mathrm{C}$. elegans nervous system. Developmental biology 263: 81-102.

Uchida, N., K. Takahashi, R. Iwasaki, R. Yamada, M. Yoshimura et al., 2018 Chemical hijacking of auxin signaling with an engineered auxin-TIR1 pair. Nat Chem Biol 14: 299-305. 
Van Wynsberghe, P. M., Z. S. Kai, K. B. Massirer, V. H. Burton, G. W. Yeo et al., 2011 LIN-28 co-transcriptionally binds primary let-7 to regulate miRNA maturation in Caenorhabditis elegans. Nat Struct Mol Biol 18: 302-308.

Vo, A. A., M. T. Levenson, J. M. Ragle and J. D. Ward, 2021 Efficient generation of a singlecopy eft-3p::TIR1::F2A:: BFP::AID*::NLS allele in the C. elegans ttTi5605 insertion site through recombination-mediated cassette exchange, pp.

Voutev, R., and E. J. Hubbard, 2008 A "FLP-Out" system for controlled gene expression in Caenorhabditis elegans. Genetics 180: 103-119.

Yesbolatova, A., Y. Saito, N. Kitamoto, H. Makino-Itou, R. Ajima et al., 2020 The auxin-inducible degron 2 technology provides sharp degradation control in yeast, mammalian cells, and mice. Nat Commun 11: 5701.

Zhang, L., J. D. Ward, Z. Cheng and A. F. Dernburg, 2015 The auxin-inducible degradation (AID) system enables versatile conditional protein depletion in C. elegans. Development 142: $4374-4384$. 
A Wild Type lin-28(ga54) B
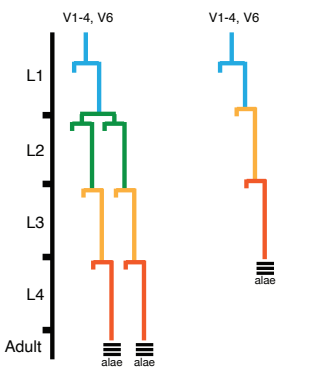

Wild Type

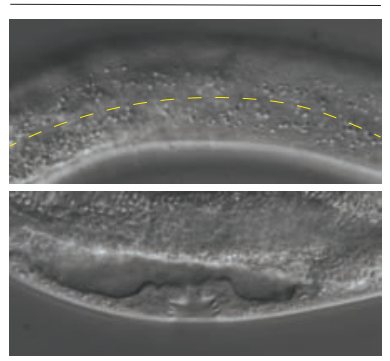

lin-28::AID*; rps-28pro::TIR1
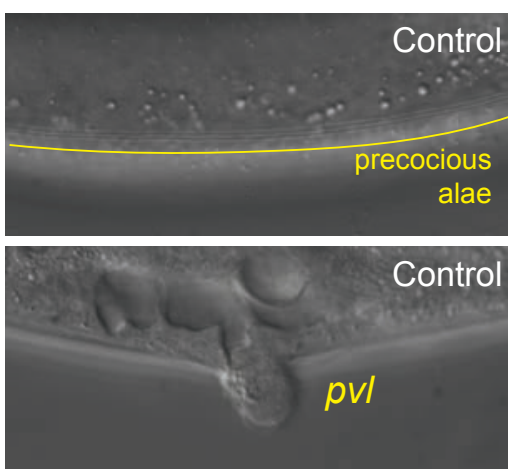

lin-28::AID*(ae157)
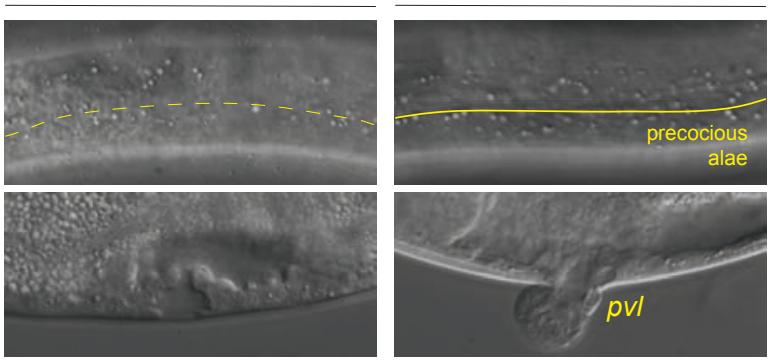

C
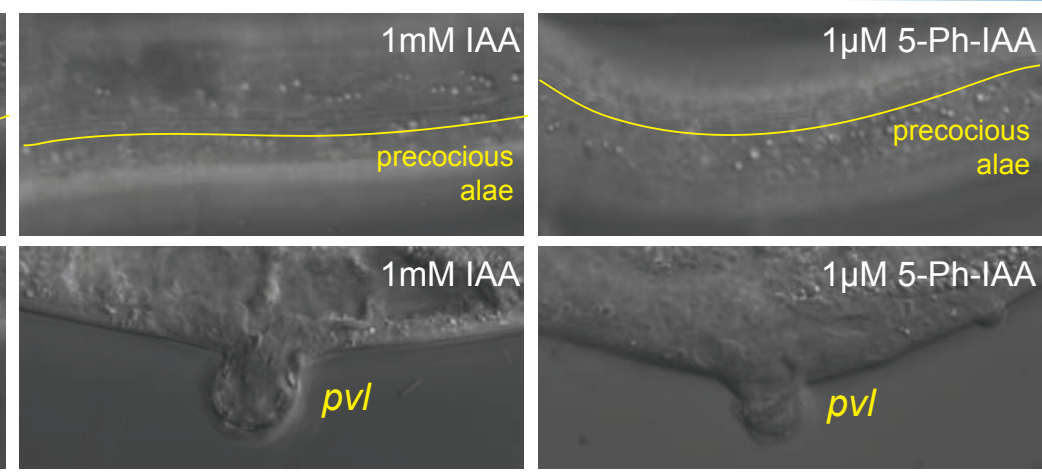

1 $\mu M$ 5-Ph-IAA

pvl

Hills-Mucky et al. 2021 Figure 1 
bioRxiv preprint doi: https://doi.org/10.1101/2021.08.06.455414; this version posted August 9, 2021. The copyright holder for this preprint (which was not certified by peer review) is the author/funder. All rights reserved. No reuse allowed without permission.

A

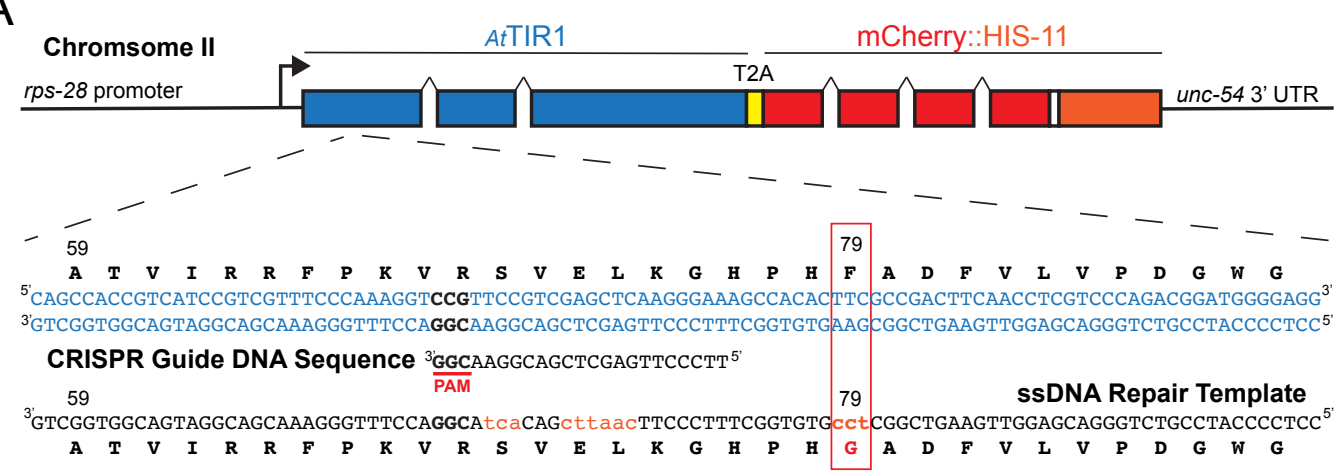

B
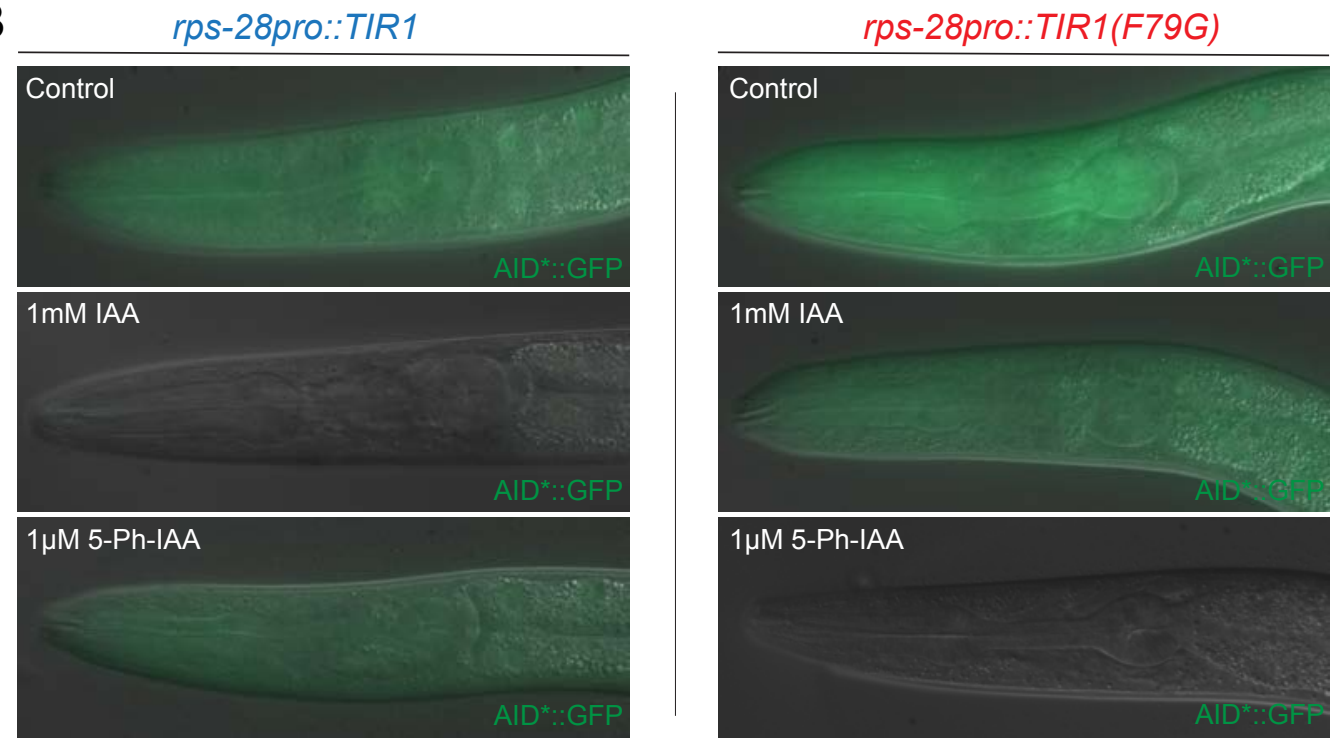

C

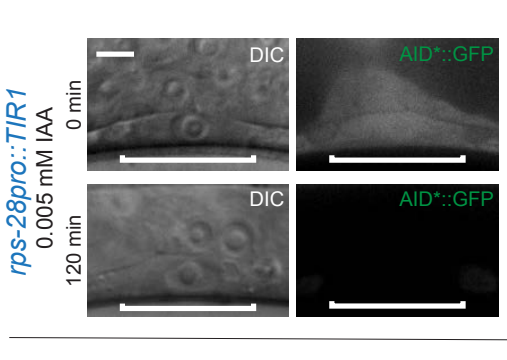

$\mathrm{D}$
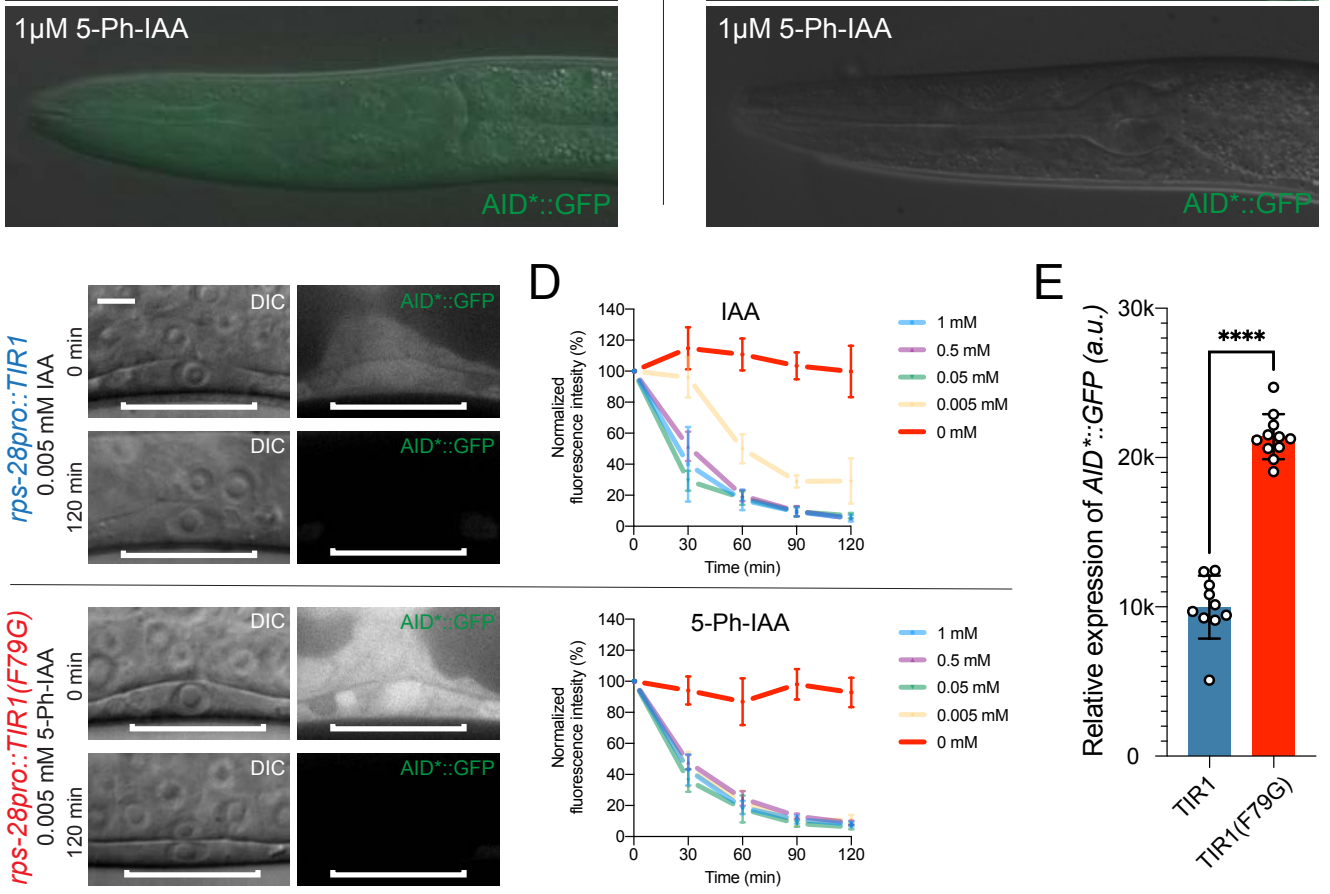

Hills-Mucky et al. 2021 Figure 2 


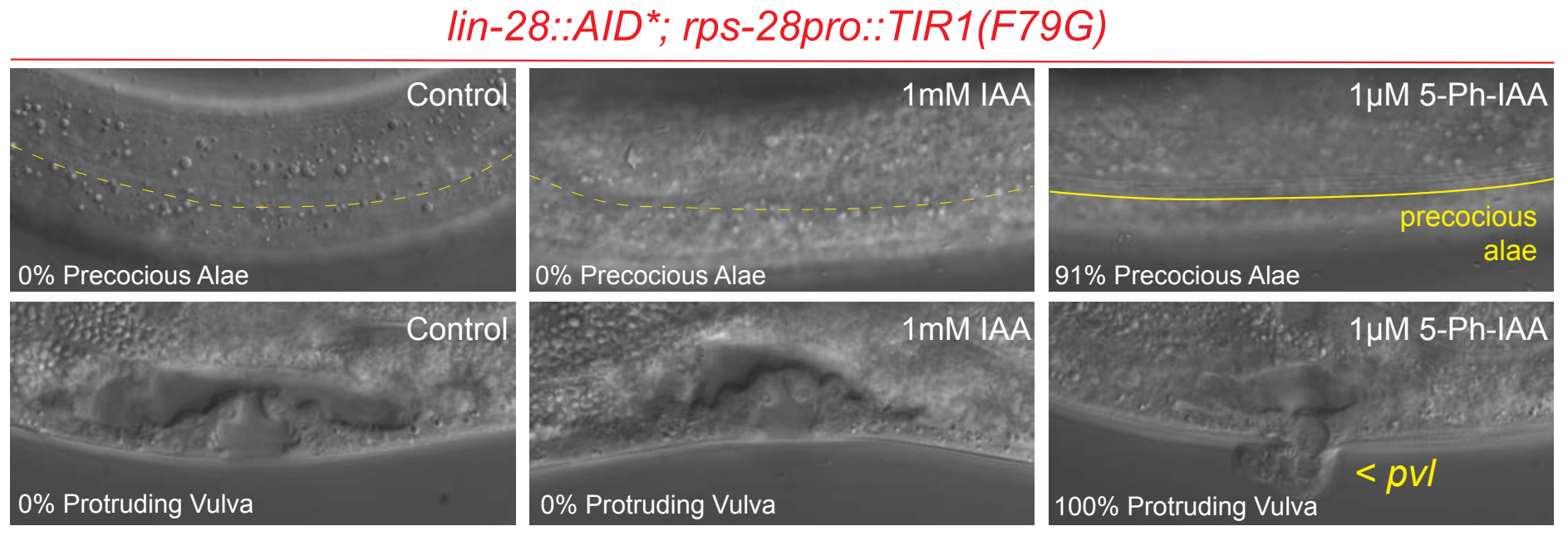

Hills-Mucky et al. 2021 Figure 3 

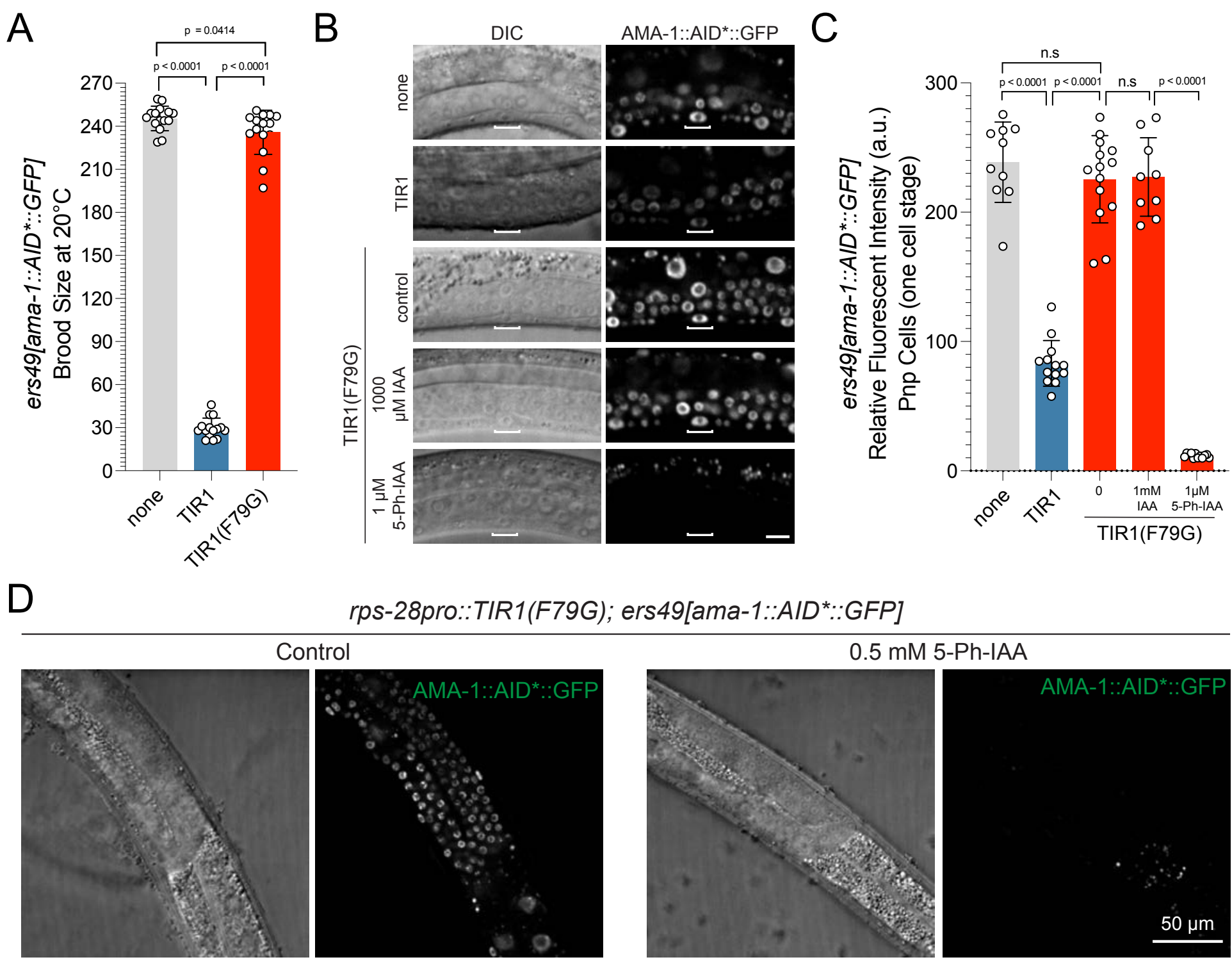

Hills-Mucky et al. 2021 Figure 4 

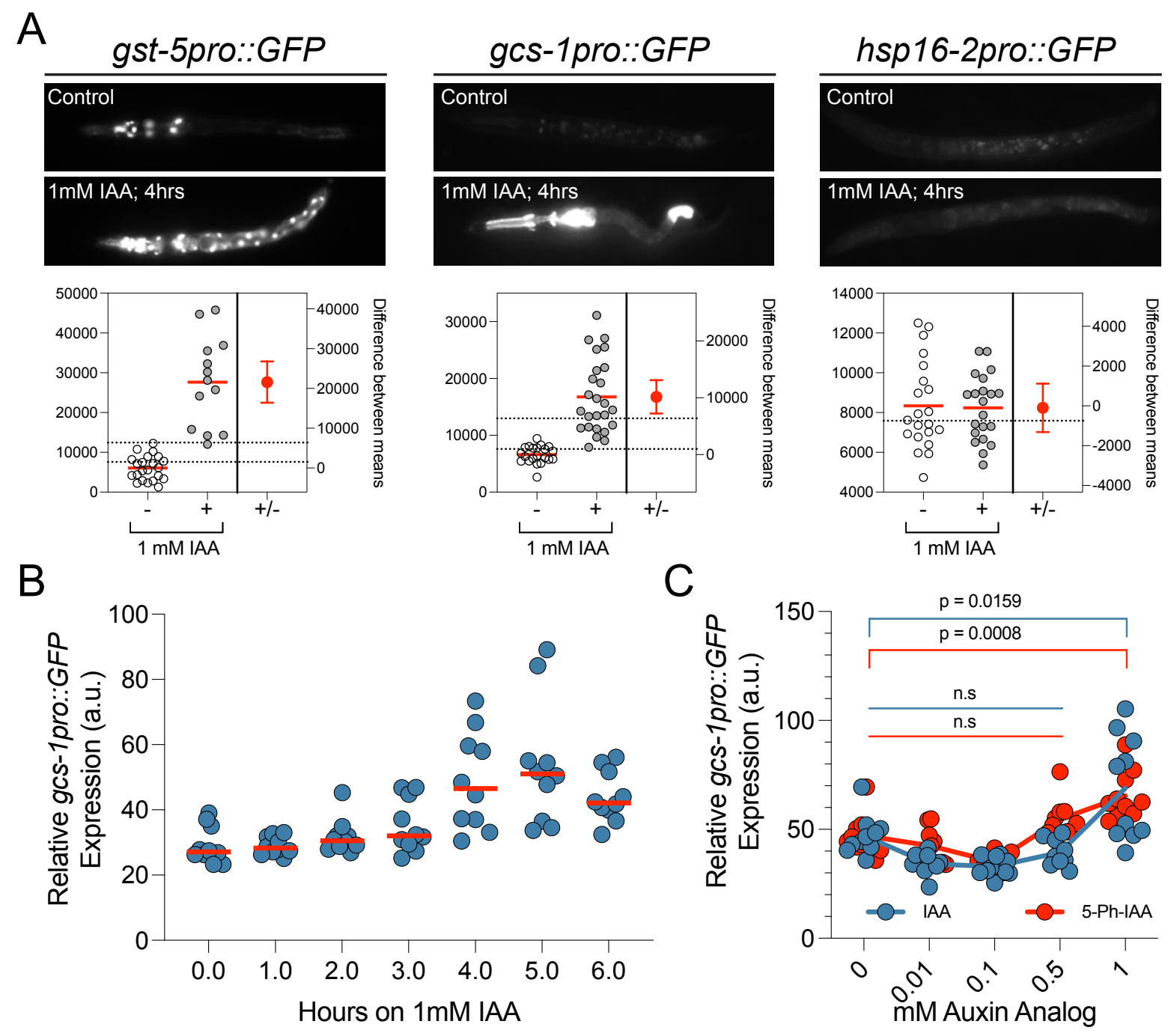

Hills-Mucky et al. 2021 Figure 5 
A

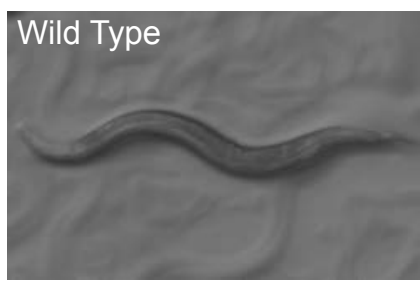

B
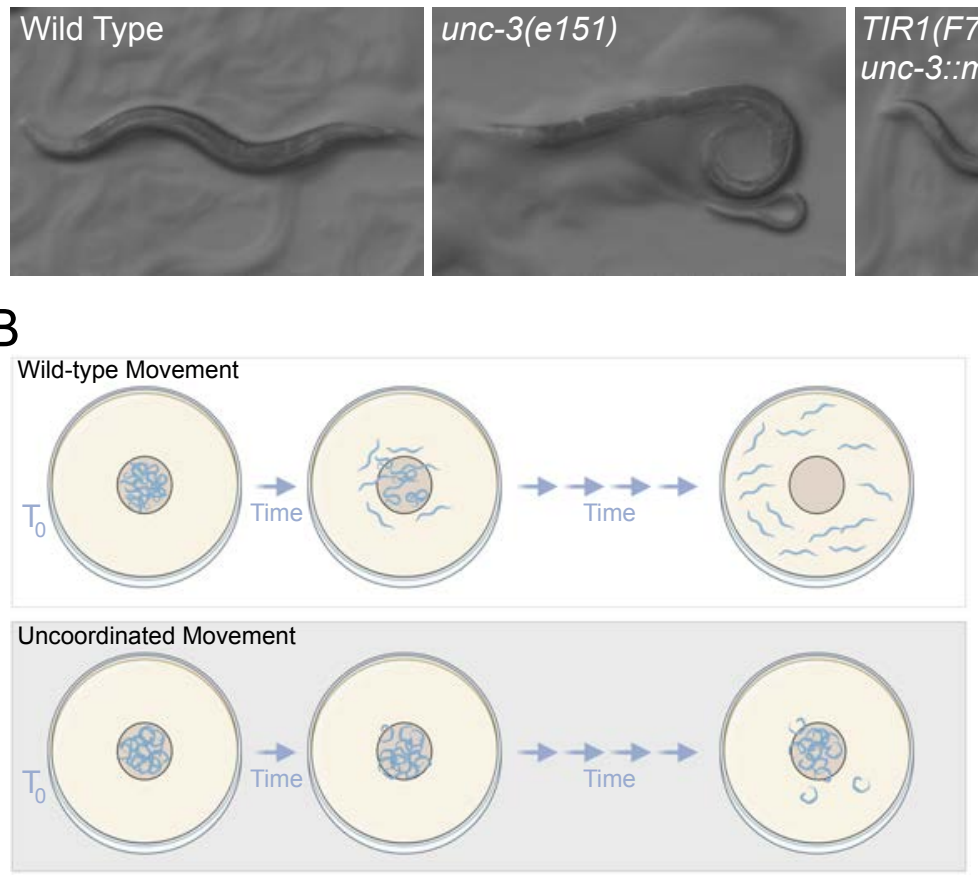
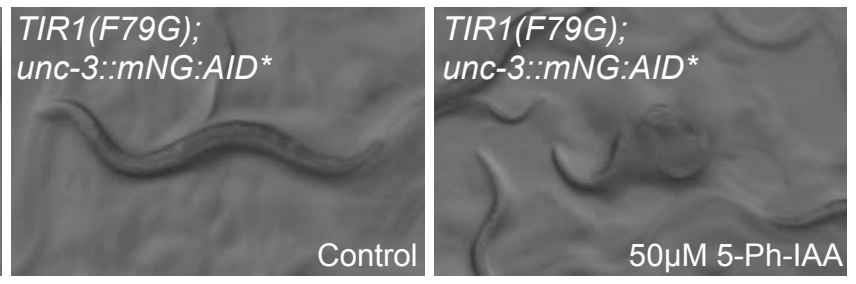

C

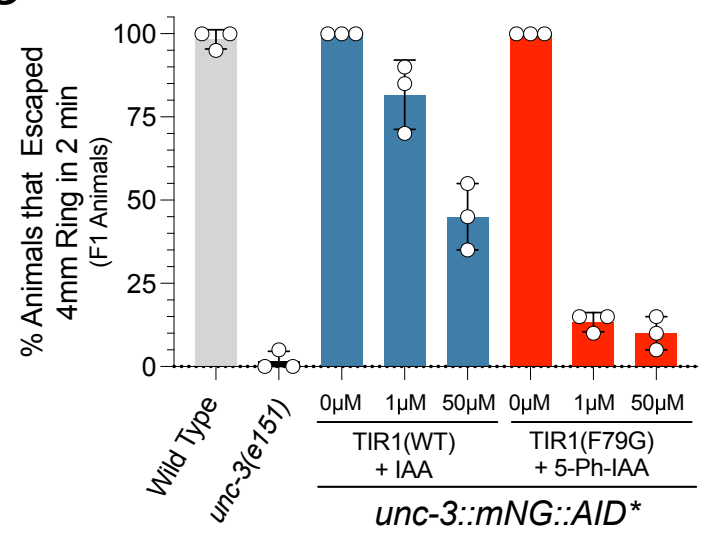

Hills-Mucky et al. 2021 Figure 6 
A

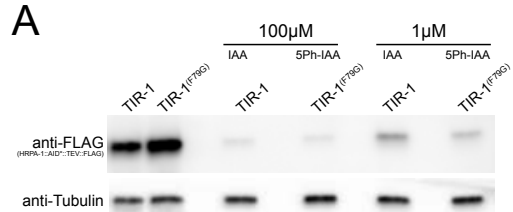

B $\quad$ C
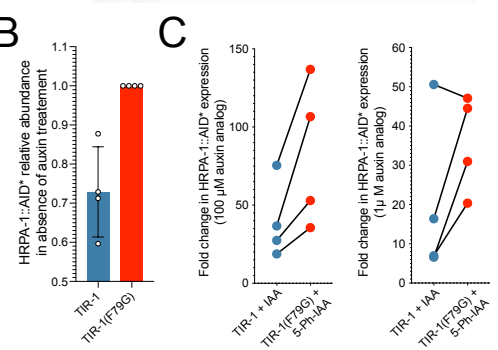

$\mathrm{D}$
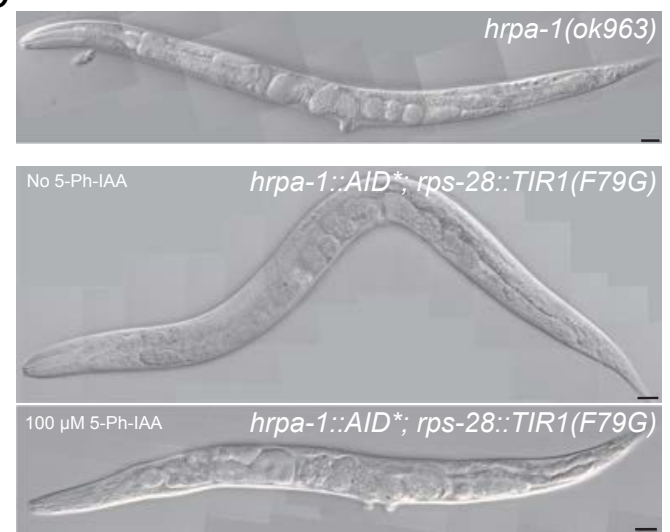

E

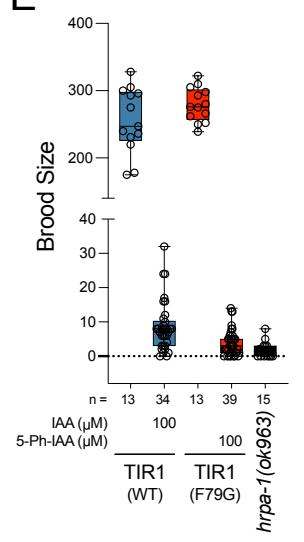

F

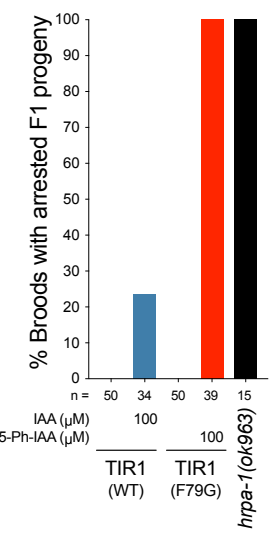

Hills-Mucky et al. 2021 Figure 7 


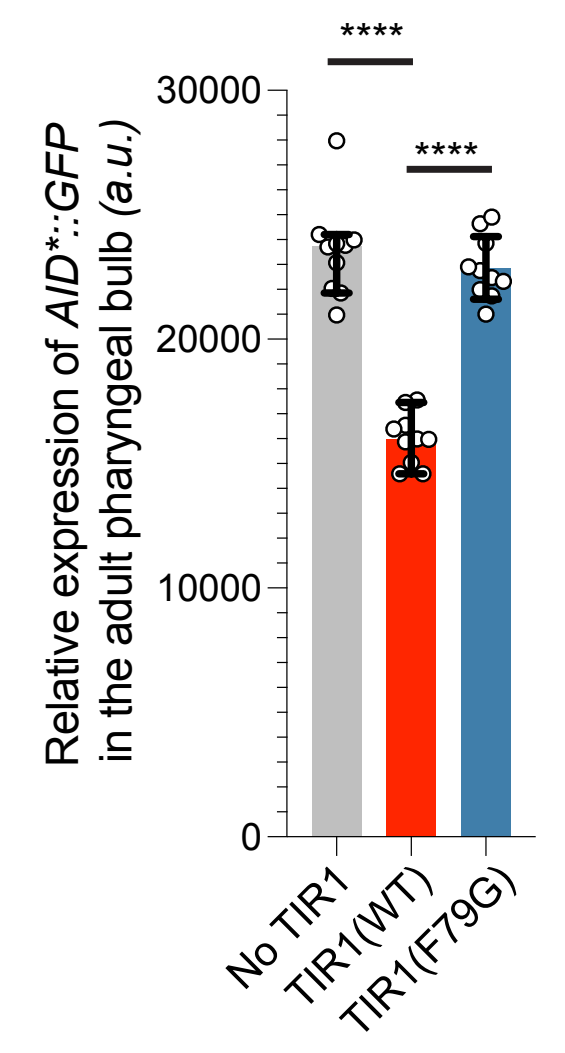

Hills-Mucky et al. 2021 Figure S1 


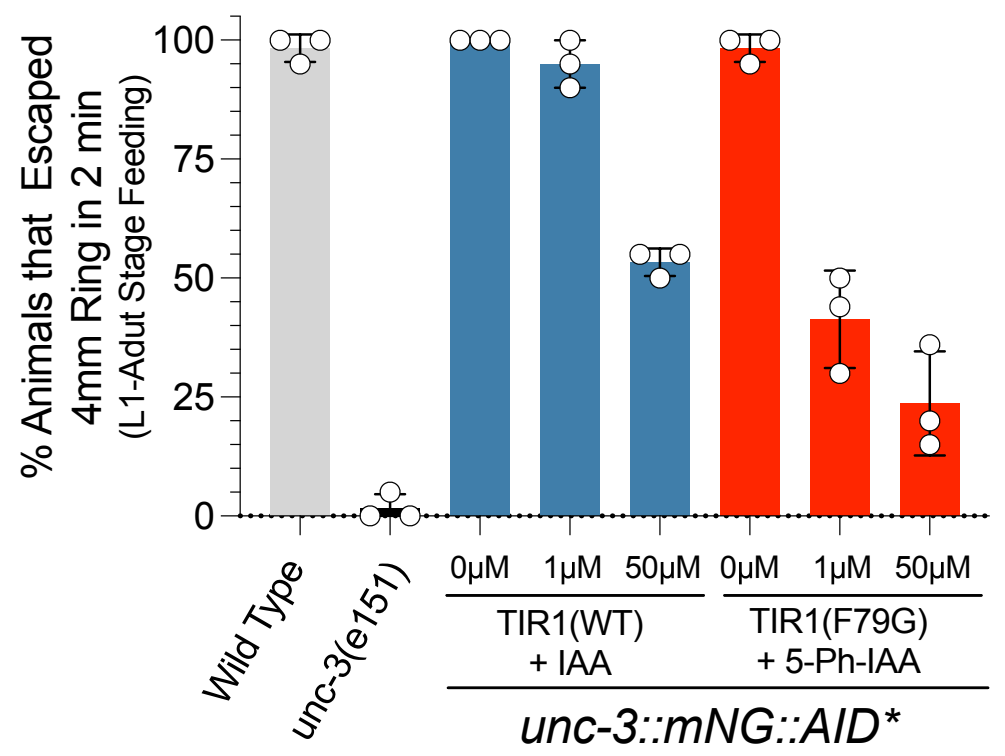

Hills-Mucky et al. 2021 Figure S2 


\section{A (Method 1) CRISPR-conversion of existing TIR1 transgene}

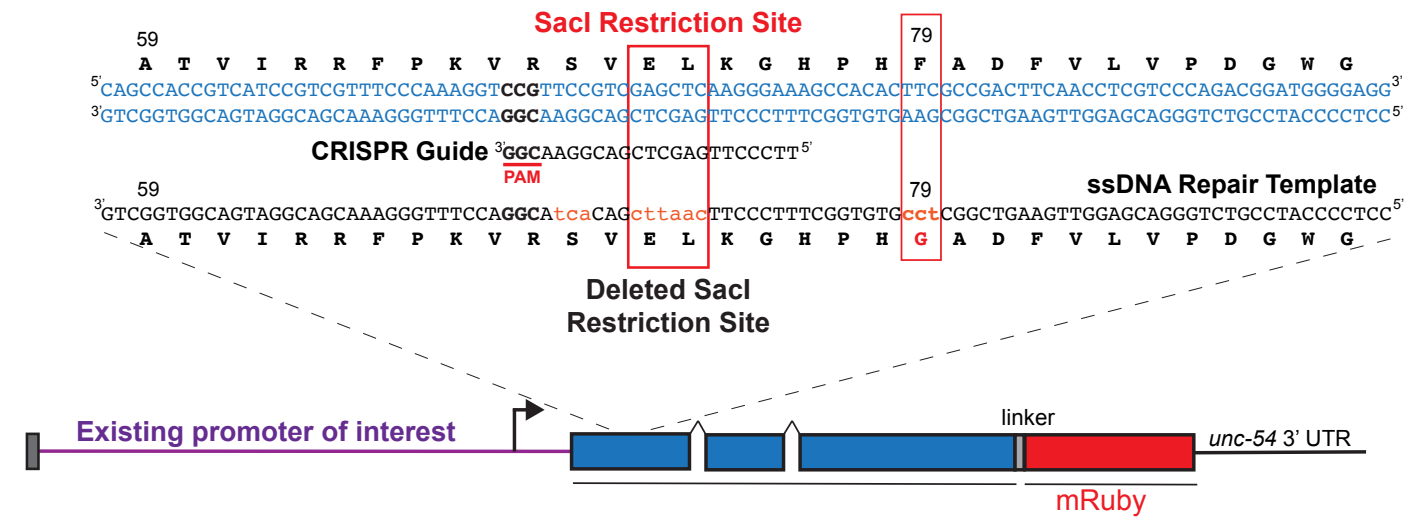

Chromsome?

\section{B (Method 2) CRISPR-conversion to replace rps-28 promoter of $c$ shls140}

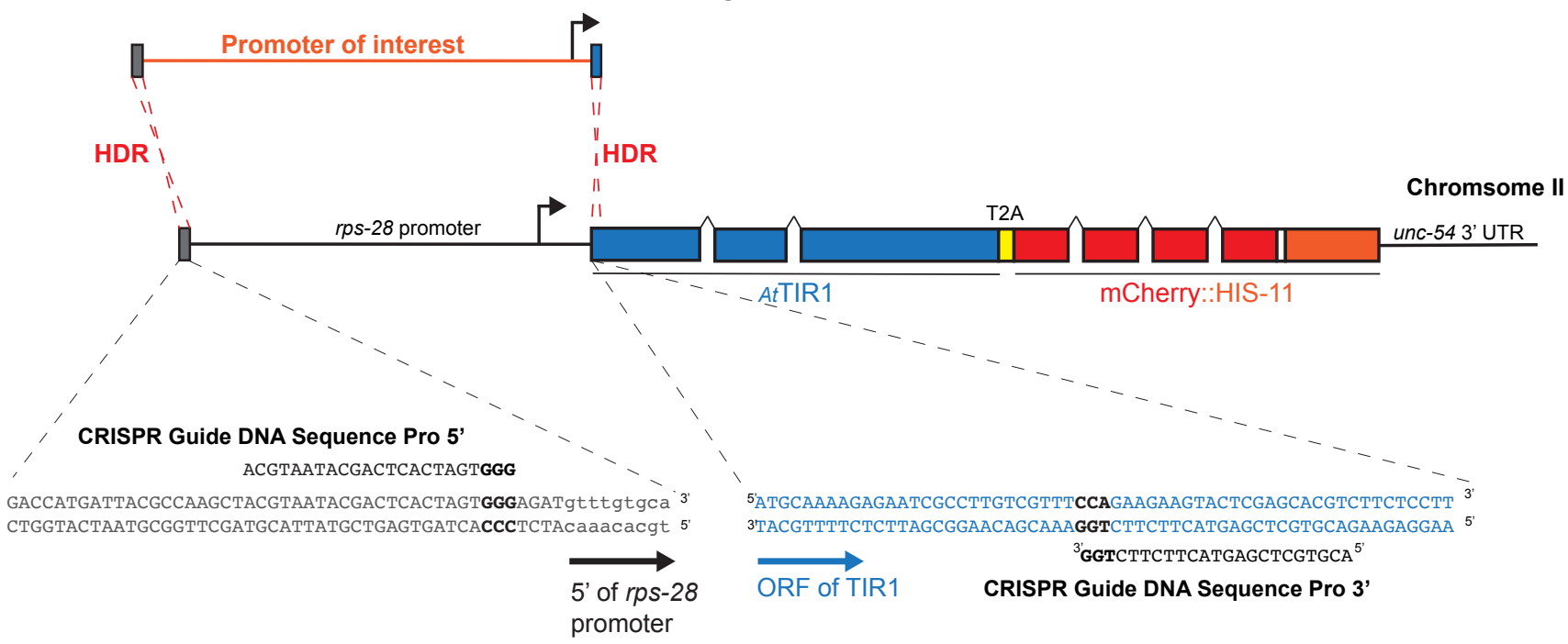

Hills-Mucky et al. 2021 Figure S3 
Table 1. Measurement of TIR1 and TIR1(F79G) dependent heterochronic phenotypes in strains harboring an AID-tagged lin-28 allele

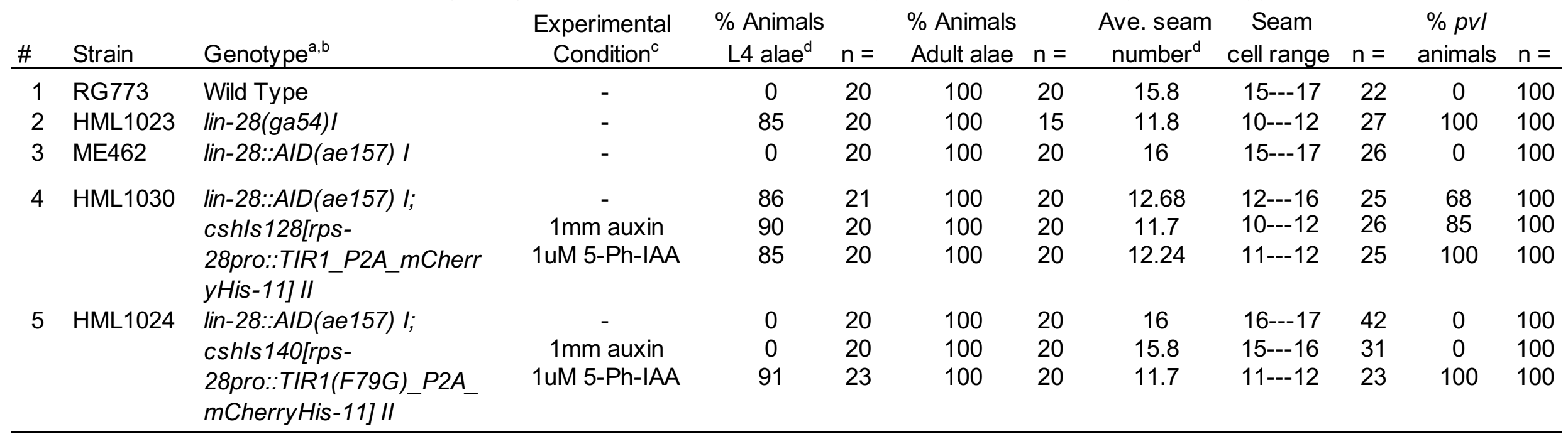

${ }^{a}$ Animals contain wIS78 V [ajm-1::GFP and scm::GFP] which were used to visualize adherence junctions and lateral seam cells.

${ }^{\mathrm{b}} \mathrm{L}$-the ae157 allele of lin-28 harbors an AID tag fused in frame to the lin-28 coding sequence generating a carboxy-terminal tagged allele.

${ }^{\mathrm{C}} \mathrm{L}$-4-staged $\mathrm{Po}$ animals were plated onto indicated plates and F1 progeny of the indicated stage were scored for

${ }^{\mathrm{d}}$ Presence and quality of cuticular alae structures were assayed by Normarski DIC optics. Only one side of each animals was scored.

${ }^{\mathrm{e}}$ Average seam cell numbers were scored by counting the number of SCM::GFP(+) cells on a single side of each animal. 


\section{Hills-Muckey et al. 2021 Strains used in this study.}

\section{Strain Genotype}

N2 wild type

HML1035 csh/s128[rps-28pro::TIR1::P2A::mCherry::HIS-11]; ieSi58 [Peft-3::degron::GFP::unc-54 3'UTR, Cbr-unc-119(+)]

Origin

HML1012 csh/s140[rps-28pro::TIR1(F79G)::P2A::mCherry::HIS-11]; ieSi58 [Peft-3::degron::GFP:::unc-54 3'UTR, Cbr-unc-119(+)]

This study

\section{VT1588 wIs79 [scm::GFP; MH27::GFP] IV}

HML1023 lin-28(ga54) I; wls79 [scm::GFP; MH27::GFP] IV

ME462 lin-28::AID(ae157); wls79 [scm::GFP; MH27::GFP] IV

HML1030 lin-28::AID(ae157) I; cshIs140[rps-28pro::TIR1_P2A_mCherryHis-11] II; wls79 [scm::GFP; MH27::GFP] IV

HML1024 lin-28::AID(ae157) I ; csh/s140[rps-28pro::TIR-1(F79G)_P2A_mCherryHis-11] II; wIs79 [scm::GFP; MH27::GFP] IV

DQM770 ama-1(ers49[ama-1::degron-GFP]) IV

DQM793 cshls140[rsp-28pro::TIR1-F79G::P2A::mCherry::his-11]; ama-1(ers49[ama-1::degron-gfp]) IV

DQM801 csh128[rsp-28pro::TIR1::P2A::mCherry::his-11)] II; unc-119(ed3); ama-1(ers49[ama-1::degron-gfp]) IV

HML817 unc-119(ed3); cshls106[unc-119; ttx-3::GFP; P(2xPCE)gst-5::GFP-pest]

LD1171 IdIs3 [gcs-1p::GFP + rol-6(su1006)]

TJ375 gp/s1 [hsp-16.2p::GFP].

HML1080 unc-3(e151)

HML1073 csh/s140[rsp-28pro::TIR1-F79G::P2A::mCherry::his-11]; unc-3(ot837)[unc-3::mNG::AID] X.

HML1075 csh128[rsp-28pro::TIR1::P2A::mCherry::his-11)] II; unc-3(ot837)[unc-3::mNG::AID] X.

UY426 (hrpa-1(ok963)/tmc25) IV

UY379 hrpa-1(zen91[HRPA-1::AID::TEV::FLAG]) IV; cshIS128 [rps-28p:TIR-1 P2A mcherry His11 unc-119(ed3)] II

UY380 hrpa-1(zen91[HRPA-1::AID::TEV::FLAG]) IV; cshIS140 [rps-28p:TIR-1(F79G) P2A mCherry His11] II

This study

This study

This study

This study

This study

This study

This study

This study

Wang et al.

2010

Mendenhall et

al. 2012

Brenner 1975

This study

This study

This study

This study

This study 